FOUNDATIONS OF COMPUTING AND DECISION SCIENCES

Vol. 42

(2017)

No. 1

DE DE GRUYTER

\title{
ROBUST AND RELIABLE PORTFOLIO OPTIMIZATION FORMULATION OF A CHANCE CONSTRAINED PROBLEM
}

\author{
Raghu Nandan SENGUPTA ${ }^{1}$, Rakesh KUMAR ${ }^{2}$
}

\begin{abstract}
We solve a linear chance constrained portfolio optimization problem using Robust Optimization (RO) method wherein financial script/asset loss return distributions are considered as extreme valued. The objective function is a convex combination of portfolio's CVaR and expected value of loss return, subject to a set of randomly perturbed chance constraints with specified probability values. The robust deterministic counterpart of the model takes the form of Second Order Cone Programming (SOCP) problem. Results from extensive simulation runs show the efficacy of our proposed models, as it helps the investor to (i) utilize extensive simulation studies to draw insights into the effect of randomness in portfolio decision making process, (ii) incorporate different risk appetite scenarios to find the optimal solutions for the financial portfolio allocation problem and (iii) compare the risk and return profiles of the investments made in both deterministic as well as in uncertain and highly volatile financial markets.
\end{abstract}

Keywords: Risk Management; Investment Analysis; Robust Optimization; Conditional Value at Risk (CVaR); Extreme Value Distribution

\section{Introduction}

The essence of modern portfolio theory is the seminal work of [60], in which one tries to maximize the expected value of the portfolio return subject to relevant constraints on portfolio variance. Another way to treat the problem would be to minimize the portfolio variance subject to constraints on the portfolio returns. In formulating the portfolio, the investor's aim is to combine financial scripts/assets in a way such that it results in diversification while at the same time maintain the required optimal risk return profile of

${ }^{1}$ Corresponding author: IME department, IIT Kanpur, Kanpur - 208016, INDIA, (Email: raghus@iitk.ac.in) and Department of Statistics and Econometrics, Faculty of Economic Sciences, University of Warsaw, 00-241 Warsaw, POLAND (Email: rsengupta@wne.uw.edu.pl)

${ }^{2}$ Ernst \& Young LLP, Gurgaon, Haryana - 122 002, INDIA. The work is a part of the master thesis work of the second author in the IME department at IIT Kanpur,

INDIA, (Email: Rakesh5.Kumar@in.ey.com) and Cell: +91-98105-31986 
the investor. The idea of diversification also highlights the relationship between financial scripts/assets which may be quantified by the concept of correlation coefficient. Let us consider this in more details. Consider an investor is interested to allocate optimal proportions/amounts of funds/money, denoted by $w_{i}, i=1, \ldots, n$, in $n$ different financial scripts/assets, with an intention to maximize the portfolio return, $\bar{r}_{P}=\sum_{i=i}^{n} \bar{r}_{i} w_{i}$, where $\bar{r}_{i}$ is the average return for the $i^{\text {th }}$ financial script/asset, $i=1, \ldots, n$. While doing so the investor has in front of him/her some realistic constraints to satisfy, which may have to do with the portfolio risk, $\sigma_{P}^{2}=\sum_{i=1}^{n} \sum_{j=1}^{n} w_{i} w_{j} \sigma_{i, j}=\sum_{i=1}^{n} \sum_{j=1}^{n} w_{i} w_{j} \rho_{i, j} \sigma_{i} \sigma_{j}$, where $\sigma_{i, j}, \sigma_{i}, \rho_{i, j}$ are covariance, standard deviation, correlation coefficient respectively, being bounded from above by a stipulated known value, $\sigma_{p}^{* 2}$, or the fact that the total investment expenses, $\sum_{i=1}^{n} I_{i}$, where $I_{i}$ is the investment expense for the $i^{t h}$ financial script, does not exceed a certain fixed percentage, $\tau_{i}$, of the total endowment value, $V_{0}$, at time $t=0$, etc. In order to obtain the desired results the investor may need to formulate and solve relevant financial optimization problems. If we pay close attention to the above problem, one would notice that the optimal proportions/amounts based on which the investor invests, may vary due to the fact that input prices of financial scripts/assets and hence, $r_{i}$, are non-deterministic. Added to this the changing economic environment may also have a fluctuating effect on $V_{0}$. It is also possible that due to extraneous considerations the changing interest rates may influence different variables like $I_{i}^{\prime} s, i=1, \ldots, n$, and $V_{0}$ considered in the problem.

Thus one observes that in trying to solve financial optimization problems or for that matter any optimization problem we encounter situations where the problem structure along with the input parameters, $\boldsymbol{D}=\left(D_{1}, \ldots, D_{n}\right)$ and/or the decision variables, $\boldsymbol{x}=$ $\left(x_{1}, \ldots, x_{n}\right)$, are non-deterministic. The question then is, how does one solve such nondeterministic problems? There are a variety of solution techniques, one of which is the concept of Robust Optimization (RO) method which we use here.

Before we discuss about RO let us consider a generic optimization model as given in (1)

$$
\begin{gathered}
\max _{\forall x} f(\boldsymbol{x}, \boldsymbol{D}) \\
\text { s.t.: } g_{j}(\boldsymbol{x}, \boldsymbol{D}) \leq 0 \\
\quad \boldsymbol{x} \in \mathbb{R}^{n}
\end{gathered}
$$

where $\boldsymbol{x} \in \mathbb{R}^{n}$ is the vector of $n$ number of decision variables, $\boldsymbol{D}$ is the parameter vector (also termed as input data), $f(\cdot)$ is the objective function and $g_{j}(\cdot)$ is the $j^{\text {th }}$ constraint where $j=1, \ldots, m$. If the parameter values are unknown then one needs to estimate or compute them accurately. Furthermore if the estimated values of the parameters differ from their nominal (average) values, then the optimal solution obtained may violate some or all of the critical constraints. This results in sub-optimal or even unfeasible solution. To tackle these uncertainties one uses the concept of Robust Optimization (RO).

Before discussing the model, the $\mathrm{RO}$ formulation with proofs and the simulation results, let us state the general plan of this research article. The focus of this paper is to model and solve linear Chance Constrained Optimization Problem (CCOP) using RO approach with application in financial portfolio optimization, the discussion of which is covered in Section 2. In Section 3 we talk about the proposed model, where we solve a robust Chance Constrained Portfolio Optimization Problem (CCPOP), with an objective function which is a convex combination of Conditional Value at Risk (CVaR) and portfolio's expected value of loss return, subject to a system of randomly perturbed chance constraints with given probabilities. The data analysis part, is then covered in Section 4, where time series return 
data of indices (considered here as financial scripts/assets) for 12 different countries, namely (i) AORD (Australia); (ii) BVSP (Brazil); (iii) CAC40 (France); (iv) DAX (Germany); (v) FTSE (England); (vi) GSPTSE (Canada); (vii) HANGSENG (Hong Kong); (viii) MERVAL (Argentina); (ix) NIKKEI (Japan); (x) NSE (India); (xi) NYSE (USA) and (xii) $S G X$ (Singapore) are considered. Finally Section 5 ends this paper with few conclusions and ideas about future research.

\section{Robust Optimization}

In real life optimization models, the parameter values are generally not known beforehand, hence one needs to estimate them from available historical data. These parameter values usually have measurement/estimation errors, and it is impossible to accurately measure them. Furthermore there may be implementation errors as it is difficult to put into practice the solution exactly as computed. RO as a technique incorporates these data uncertainties such that the solution found does not violate the critical constraints for all or most possible realizations of the uncertain parameters. The levels of uncertainty in the parameter/coefficient values are described using uncertainty sets and these uncertainty sets contain all possible values that may be realized for the uncertain parameters. Using these uncertainty sets we convert the uncertain optimization problem into its deterministic counterpart, and in doing so it must be remembered that the optimal solution for the problem should remain feasible for any realization of the uncertain parameters within the pre-specified uncertainty sets of these parameters. Thus the corresponding RO formulation for the optimization problem, (1), (considering uncertainties in $\boldsymbol{D}$ ) may be expressed as shown in (2).

$$
\begin{gathered}
\max _{\forall x} t \\
\text { s.t.: } \operatorname{Pr}\{f(\boldsymbol{D}, \boldsymbol{x}) \geq t\} \geq \beta_{0} \\
\operatorname{Pr}\left\{g_{j}(\boldsymbol{D}, \boldsymbol{x}) \leq 0\right\} \geq \beta_{j}, j=1, \ldots, m \\
\boldsymbol{x} \in \mathbb{R}^{n}
\end{gathered}
$$

where, $\beta_{0}$, is the level of probability associated with the objective function, $\beta_{j}^{\prime} s$ are the probability levels corresponding to the $j^{\text {th }}$ constraint, $j=1, \ldots, m$, while $t$ is the equivalent RO objective which needs to be optimized. Though (2), seems expressively rich but there are some computational difficulties. For example, it is difficult to obtain the true distribution of the uncertain parameters. Furthermore even if we know the distributions, the evaluations of chance constraints are computationally challenging, let alone the optimization of the model. Moreover the presence of chance constraint can hamper the desired convexity properties and hence enhance the complexity of the problem.

Thus in RO the focus is to design and solve optimization problems keeping in mind the non-deterministic nature of the parameters/variables. As an optimization technique RO is relatively new and is based on the idea that the non-deterministic nature of parameters/variables may be described using the concepts of uncertainty sets. In RO our aim is to obtain solutions such that they are robust up to the permissible perturbation of the probabilistic values of parameters/variables. Furthermore, one may allow fluctuation in the parameters/variables values in order to study the sensitivity of the final results, as well as allow acceptable deviation of the objective function(s). Thus RO provides us a different approach to handle data uncertainty in optimization model by proposing the robust 
counterpart approach. In order to capture the effect of uncertainty of the parameters/variables on the feasibility and optimality of the solutions, we form uncertainty sets such that these sets contain all possible values that may be realized for the uncertain parameters/variables. The concept of uncertainty sets, is used to model the perturbation of the uncertain parameter by utilizing the idea of nominal value/average value along with it corresponding fluctuations, such that the variations in the fluctuations are considered keeping in mind the level of reliability one wants to ensure. Ideas of $L_{2}$ and $L_{\infty}$ norms are used, utilizing the box and ball robustness of the uncertainty sets, such that all the probable fluctuations in the parameter values are thereby considered. In doing so the corresponding robust value as specified in the model is incorporated using concepts of standard normal deviate, $\Phi$, such that it is incorporated into the problem formulation through the concept of perturbation. One may refer to [7], [15] and [22] to get a good idea about the concept of uncertainty sets, perturbations, and the different type of uncertainty sets used in RO.

The work by [68] is considered as the first paper in RO. As an optimization method it has been revitalized in the late 1990s and early 2000s through the seminal work of [17], [18], [27] and [47]. Few good survey papers in RO and related areas of uncertainty are those by [28], [39], [41] and [67]. Good works in the area of RO and its use in methods as diverse as multi-stage programming, cone programming, linear optimization, convex programming, semi-definite programming, etc., are those by [2], [6], [12], [13], [14], [16], [19], [21], [24], [25], [26], [29], [31], [37], [38], [43], [44], [47] and [62]. Considering its usefulness, RO has also been adopted in many application areas across various fields like optimal control theory, circuit design, engineering, statistics [5], [11], [35], [36], [46], and [70]), inventory management, dynamic pricing and revenue management [1], [8] and [30], network with multi commodity flow [3], etc. In the domain of portfolio optimization, asset allocation problem, financial risk management, etc., few good references are [10], [33], [40], [48], [49], [50], [51], [52], [54], [61], [63], [69] and [71]. RO has also been used in the context of dynamic models and few relevant references for this are [20], [45] and [55]. Few interesting readings which one may refer to get a good understanding of $\mathrm{RO}$ and its similarities as well as differences with stochastic programming and other such nondeterministic methods are, [34], [42], [56], [64] and [65]. Other interesting writings like [4] (use of RO for maximizing payoff functions under ambiguous distribution), [9] and [53], (solutions methods for different class of RO formulations), etc., may also be referred by the readers to get a good idea about RO as an useful method for optimization. Finally few good books, survey papers, application tools, etc., for RO are by [7], [15], [23], [32], [57] and [66].

\section{Problem Description and Model Formulation}

In our proposed model we simultaneously minimize CVaR as well as maximize expected value of loss return, subject to two probability constraints whereby uncertainty is present in one of the parameters, which is loss return of each of the $N$ number of indices/financial scripts/assets considered in the portfolio. It should be remembered that no uncertainty exists for the objective function value. Before discussing the model, we explain the variables used in this model, for a better understanding of the model amongst readers. A variable may be classified as an element of $\boldsymbol{D}$ or $\boldsymbol{x}$ or as deterministic. For the case of $\boldsymbol{D}$ or $\boldsymbol{x}$, the variable has been marked accordingly, while for the one which is deterministic we have not labeled 
it and have left that for the reader to understand and appreciate its significance with respect to the problem.

1) $\quad r_{j, i}(\in \boldsymbol{D})$ : The loss return of the $j^{t h}$ index $(j=1, \ldots \ldots, N)$ for the $i^{t h}$ day $(i=1, \ldots \ldots, T)$.

2) $\quad \bar{r}_{j}(\in \boldsymbol{D})$ : The nominal value of loss return of the $j^{\text {th }}$ index $(j=1, \ldots \ldots, N)$.

3) $\sigma_{j}^{2}(\in \boldsymbol{D})$ : The variance of loss return of the $j^{t h}$ index $(j=1, \ldots \ldots, N)$.

4) $\quad x_{j}(\in \boldsymbol{x})$ : The weight of investment in the $j^{t h}$ index, $(j=1, \ldots \ldots, N)$.

5) $\quad N$ : The number of different indices/financial scripts/assets considered in the portfolio (denoted by $P$ ).

6) $\quad T$ : The total time period for which we consider the price of each of the $j^{\text {th }}$ index, $(j=1, \ldots \ldots, N)$.

7) $\alpha_{\Delta L}$ : The confidence level for $\operatorname{VaR}_{P, \Delta L}$, considering the loss $(\Delta L)$ distribution of the portfolio.

8) $\quad \beta_{1}$ and $\beta_{2}$ : The probability level values corresponding to the first and the second constraints, respectively.

9) $\gamma_{\Delta L, P}$ : The variable $(\in \mathbb{R})$ over which the minimization of $\operatorname{CVaR}_{P, \Delta L}$ is done, considering loss $(\Delta L)$ distribution of the portfolio.

10) $\quad \operatorname{VVaR}_{P, \Delta L}$ : Conditional Value at Risk of the portfolio's loss distribution that needs to be minimized.

11) $r_{P, \Delta L}(\in \boldsymbol{D})$ : Return of the portfolio's loss distribution that needs to be maximized.

12) $\Delta L(\in \boldsymbol{D})$ : Loss distribution for the portfolio, $P$.

13) $\lambda_{1}$, and $\lambda_{2}$ : The variables, $\in[0,1]$, which are required to ensure a convex combination of $C V a R_{P, \Delta L}$ and $r_{P, \Delta L}$ of the portfolio.

14) $\quad Z(\in \boldsymbol{D})$ : Uncertainty set for the robust counterpart.

15) $\zeta_{j}(\in \boldsymbol{D})$ : Perturbation vector set for $j=1, \ldots \ldots, N$.

16) $\Omega_{1}$ and $\Omega_{2}(\in \boldsymbol{D})$ : Radius of ball or ellipsoid for the first and the second constraint respectively.

17) $z_{j}(\in \boldsymbol{D})$ : Dummy variables for the first robust counterpart, $j=1, \ldots \ldots, N$.

18) $w_{j}(\in \boldsymbol{D})$ : Dummy variables for the second robust counterpart, $j=1, \ldots \ldots, N$.

Based on the above set of information, the model is of the form as shown in (3).

$$
\begin{gathered}
\min _{\forall x}\left[\lambda_{1} \operatorname{CVaR} R_{P, \Delta L}-\lambda_{2} r_{P, \Delta L}\right] \\
\text { s.t. } \operatorname{Pr}\left[\left\{\frac{1}{\left(1-\alpha_{\Delta L}\right) T} \sum_{i=1}^{T} \sum_{j=1}^{N}\left(r_{j, i} x_{j}-\gamma_{\Delta L, P}\right)^{+}+\gamma_{\Delta L, P}\right\} \leq C V a R_{P, \Delta L}\right] \geq \beta_{1} \\
\operatorname{Pr}\left[\left\{\frac{1}{T} \sum_{i=1}^{T} \sum_{j=1}^{N} r_{j, i} x_{j}\right\} \geq r_{P, \Delta L}\right] \geq \beta_{2} \\
\sum_{j=1}^{N} x_{j}=1 \\
\sum_{k=1}^{2} \lambda_{k}=1 \\
x_{j} \geq 0, \quad \gamma_{\Delta L, P} \in \mathbb{R}, j=1, \ldots, N
\end{gathered}
$$

The objective function comprises of two parts. The first is an unknown value of conditional value at risk, $C V a R_{P, \Delta L}$, which we intend to minimize while the second part is the unknown value of expected loss return, $r_{P, \Delta L}$, which we need to maximize. The first constraint denotes that the conditional value at risk of the loss return distribution for the 
portfolio, i.e., $\left\{\frac{1}{\left(1-\alpha_{\Delta L}\right) T} \sum_{i=1}^{T} \sum_{j=1}^{N}\left(r_{j, i} x_{j}-\gamma_{\Delta L, P}\right)^{+}+\gamma_{\Delta L, P}\right\}$, is less than or equal to the objective $\operatorname{CVaR}_{P, \Delta L}$ value with a level of probability $\beta_{1}$. On the other hand the second constraint implies that the return of the portfolio's loss distribution, $\left\{\frac{1}{T} \sum_{i=1}^{T} \sum_{j=1}^{N} r_{j, i} x_{j}\right\}$, is greater than or equal to the objective return value, $r_{P, \Delta L}$, with $\beta_{2}$ as its corresponding probability. The third constraint implies that the sum of the weights of all investments should equal to one, while the fourth constraint ensures the convex property of the objective function. Finally the fifth constraint imposes the condition that short-selling is not allowed for any of the indices/financial scripts/assets as well as the fact that conditional value at risk can assume any real number.

Theorem 1: The robust counterpart of the uncertain linear constraint $\operatorname{Pr}\left[\left\{\frac{1}{\left(1-\alpha_{\Delta L}\right) T} \sum_{i=1}^{T} \sum_{j=1}^{N}\left(r_{j, i} x_{j}-\gamma_{\Delta L, P}\right)^{+}+\gamma_{\Delta L, P}\right\} \leq C V a R_{P, \Delta L}\right] \geq \beta_{1}$ with uncertainty set $Z=\left\{\zeta_{j} \in \mathbb{R}^{N}:\|\zeta\|_{\infty} \leq 1,\|\zeta\|_{2} \leq \Omega_{1}\right\}=$ Box $_{1} \cap$ Ball $_{\Omega_{1}}$ is equivalent to the system of conic quadratic constraints

$$
\begin{gathered}
z_{j}+w_{j}=\sum_{j=1}^{N}\left[\sigma_{j}\right]^{T} x_{j}, j=1, \ldots, N \\
{\left[\left\{\frac{1}{\left(1-\alpha_{\Delta L}\right) T} \sum_{i=1}^{T} \sum_{j=1}^{N}\left(\bar{r}_{j} x_{j}+\sum_{N}\left|z_{j}\right|+\Omega_{1} \sqrt{\sum_{j=1}^{N} w_{j}^{2}}-\gamma_{\Delta L, P}\right)^{+}\right\}+\gamma_{\Delta L, P}\right] \leq \operatorname{CVaR}_{P, \Delta L}}
\end{gathered}
$$

Here $x_{j}$ components of every feasible solutions to these equations (4) and (5) satisfy the randomly perturbed inequality, with probability at least $\left\{1-\exp \left(-\frac{\Omega_{1}^{2}}{2}\right)\right\}$, where $z_{j}$ and $w_{j}$ are the dummy variables for box and ball robustness respectively.

Proof: See Appendix A.1 for proof.

Theorem 2: The robust counterpart of the uncertain linear constraint $\operatorname{Pr}\left[\left\{\frac{1}{T} \sum_{i=1}^{T} \sum_{j=1}^{N} r_{j, i} x_{j}\right\} \geq r_{P, \Delta L}\right] \geq \beta_{2} \quad$ with uncertainty set $z=\left\{\zeta \in \mathbb{R}^{N}:\|\zeta\|_{\infty} \leq\right.$ $\left.1,\|\zeta\|_{2} \leq \Omega_{2}\right\}=\operatorname{Box}_{1} \cap$ Ball $_{\Omega_{2}}$ is equivalent to the system of conic quadratic constraints

$$
\begin{array}{r}
z_{j}+w_{j}=\sum_{j=1}^{N}\left[\sigma_{j}\right]^{T} x_{j}, j=1, \ldots \ldots \ldots, N \\
{\left[\frac{1}{T}\left(\sum_{i=1}^{T} \sum_{j=1}^{N} \bar{r}_{j} x_{j}-\sum_{N}\left|z_{j}\right|-\Omega_{2} \sqrt{\sum_{j=1}^{N} w_{j}^{2}}\right)\right] \geq r_{P, \Delta L}}
\end{array}
$$

Here $x_{j}$ components of every feasible solution to these equations (6) and (7) satisfy the randomly perturbed inequality, with probability at least $\left\{1-\exp \left(-\frac{\Omega_{2}^{2}}{2}\right)\right\}$; where $z_{j}$ and $w_{j}$ are the dummy variables for box and ball robustness respectively.

Proof: See Appendix A.2 for proof.

Using the above two theorems (Theorem 1 and Theorem 2), the robust counterpart of the model is as follows, (8). 


$$
\begin{gathered}
\min _{x}\left[\lambda_{1} \times \operatorname{CVaR}_{P, \Delta L}-\lambda_{2} \times r_{P, \Delta L}\right] \\
\text { s.t. }\left[\left\{\frac{1}{\left(1-\alpha_{\Delta L}\right) T} \sum_{i=1}^{T} \sum_{j=1}^{N}\left(\bar{r}_{j} x_{j}+\sum_{N}\left|z_{j}\right|+\Omega_{1} \sqrt{\sum_{j=1}^{N} w_{j}^{2}}-\gamma_{\Delta L, P}\right)^{+}\right\}+\gamma_{\Delta L, P}\right] \leq \operatorname{CVaR}_{P, \Delta L} \\
{\left[\frac{1}{T}\left(\sum_{i=1}^{T} \sum_{j=1}^{N} \bar{r}_{j} x_{j}-\sum_{N}\left|z_{j}\right|-\Omega_{2} \sqrt{\sum_{j=1}^{N} w_{j}^{2}}\right)\right] \geq r_{P, \Delta L}} \\
z_{j}+w_{j}=\sum_{j=1}^{N}\left[\sigma_{j}\right]^{T} x_{j}, j=1, \ldots \ldots \ldots, N \\
\sum_{j=1}^{N} x_{j}=1 \\
\sum_{i=1}^{2} \lambda_{i}=1 \\
x_{j} \geq 0, \quad \gamma_{\Delta L, P} \in \mathbb{R}, j=1, \ldots, N
\end{gathered}
$$

\section{Data Description, Pre-processing and Results with Discussions}

\subsection{Data Description and Pre-processing}

The dataset for the computational analysis comprises of the daily closing prices of indices (considered here as financial scripts/assets) from 12 countries as mentioned in Section 1. The data series for each of the indices/financial scripts/assets is considered for a range of 11 years, which corresponds to a total number of 2840 trading days. For the simulation study we divide each of the data series into two different sets, each of size 1420 , where the first set of 1420 return data points, which is from 01/01/2000 (dd/mm/yyyy) to 31/12/2005 (dd/mm/yyyy) comprise the in-sample data series. While the data series from 01/01/2006 (dd/mm/yyyy) to 31/12/2010 (dd/mm/yyyy) is considered as the out-of-sample dataset.

Use of fat tailed distributions for financial modeling is very important. Hence to ascertain that the loss distributions of asset returns are in general heavy tailed (i.e., EVD) we conduct relevant statistical tests. Apart from that we also calculate the parameters of the underlying loss distribution of returns for all the 12 indices (considered here as financial scripts/assets), using which we generate data for both in-sample as well as out-of-sample simulation tests. Our first job is to obtain the loss distribution of returns which we do by considering only those values of returns which are negative. Next we consider nonoverlapping sets of these negative values and consider the maximum negative value of each such set as a candidate for the block bootstrap method ([58], [59]). A sample of 71 data points are used whereby each point is the maximum negative loss value pertaining to a block of 20 consecutive negative return data points. Thus with these 71 values we perform block-bootstrap sampling. It should be noted that for the time series data of negative returns one simply cannot use bootstrap method because the empirical distribution does not include any of the time series information as required. Hence one has to take recourse of block bootstrap method, whereby the time series is re-sampled in blocks keeping in mind that the blocks are sufficiently long. This ensures that each block preserves, in the re-sampled series, the dependence structure present in the original data sequence. Thus the re-sampling scheme implies that we sample with replacement from the blocks and form the bootstrap time series estimates of the parameters which are of relevance to us. In the context of our research, we denote the $b^{\text {th }}(b=1, \ldots, B=5000)$ bootstrap estimate of $r_{j}, j=1, \ldots, N=$ 12 as $\hat{r}_{j, b}$, and utilize these block bootstrap values to calculate the estimates of the means as 
well as that of the variances of all the 12 indices/financial scripts/assets. Using these means (i.e., location parameters) and variances (i.e., scale parameters) for both in-sample as well as out-of-sample data sets we generate the random extreme value numbers for the in-sample and out-of-sample data series respectively. These in-sample and out-of-sample data series thus generated can now be considered as the population of the negative return distributions of all the 12 indices/financial scripts/assets included for our study. We perform Q-Q plots of the block boot-strap results and draw the density function for all the 12 indices/financial scripts/assets to check the underlying sample distributions of the indices/financial scripts/assets. Lilliefors tests are also performed to check the hypothesis whether the return of all the different indices/financial scripts/assets as well as the bootstrapped expected returns (i.e., mean values) come from normal distribution. From the tests (both in-sample as well as out-of-sample) it is apparent that none of the 12 indices/financial scripts/assets follow normal distribution, but on the contrary are closer to EVD, such that each has heavy tails. For paucity of space we do not show these detailed results/graphs for Q-Q plots, density functions and Lilliefors tests, as our main emphasis is to discuss the simulations results for the RO model.

To compare our proposed probabilistic model we simulate our model for different values of probability levels $\beta_{1}=\beta_{2}$ and confidence levels of $\alpha_{\Delta L}$. We then evaluate the values of $\operatorname{CVaR}_{P, \Delta L}, \operatorname{VaR}_{P, \Delta L}$ and $r_{P, \Delta L}$ thus obtained with the corresponding values generated using the deterministic equal weight and deterministic unequal weight models. For the reader we would like to clarify that the deterministic equal weight example signifies the case where contributions of each country's index/financial script/asset in the portfolio is equal, along with the fact that prices are known with certainty. On the other hand the deterministic unequal weight method denotes the instance where the optimization model calculates the contribution of each country's index/financial script/asset in the final portfolio, while still considering price of indices/financial scripts/assets as deterministic. The runs for the computational analysis are performed using MATLAB 7.8.0, on a $2.0 \mathrm{GHz}$ Pentium 4 machine and the operating system used is Windows Vista for MATLAB codes. Along with our developed MATLAB codes we also take the help of Robust Optimization Made Easy (ROME) (URL: www.robustopt.com) modelling language developed by Melvyn Sim and Joel Goh ([66]). Moreover MOSEK (URL: www.mosek.com) is also utilized as the optimization solver engine to handle the computational aspect of second order cones. Finally all the different analyses discussed, are run for both in-sample as well as for out-of-sample data sets.

\subsection{Results with Discussion}

In this section we discuss the simulated optimization results for both in-sample and out-ofsample data sets wherein the changes in portfolio risk (measured using $V_{a} R_{P, \Delta L}$ and $\left.\operatorname{CVaR}_{P, \Delta L}\right)$ as well as portfolio return values $\left(r_{P, \Delta L}\right)$ are observed with respect to the changing risk appetite levels ( $\alpha$ and $\beta$ values) of the investor. In order to study the impact of uncertainty on stock-prices and hence on risk-return profile of an investor we also compare the results obtained in the uncertain scenario with those found in the deterministic cases. We run our model for different levels of $\alpha_{\Delta L}$ (i.e., 90\%, 95\%, 97\% and 99\%) and $\beta_{1}=\beta_{2}$ (i.e., 0.90, 0.95, 0.97 and 0.99) values. Based on 2497 number of runs for each of the combinations, we report the values of $\overline{C V a R}_{P, \Delta L}, S D\left(C V a R_{P, \Delta L}\right), \bar{r}_{P, \Delta L}$ and $S D\left(r_{P, \Delta L}\right)$ 
obtained for both in-sample as well as for out-of-sample data series separately. The summary of these results are shown in tables $1 \& 2$.

Table 1. Average and Standard deviation values of $C \operatorname{VaR}_{P, \Delta L}$ and $r_{P, \Delta L}$ for the insample data set

\begin{tabular}{|c|c|c|c|c|c|c|}
\hline \multicolumn{7}{|c|}{$\alpha_{\Delta L}=0.90$ and $V a R_{P, \Delta L}=-0.04500$} \\
\hline \multicolumn{2}{|c|}{ Deterministic equal weights } & \multicolumn{2}{|c|}{ Deterministic un-equal weights } & \multirow{2}{*}{$\begin{array}{l}\beta_{1} \\
= \\
\beta_{2}\end{array}$} & \multicolumn{2}{|c|}{$\begin{array}{l}\text { Probabilistic unequal weights } \\
\end{array}$} \\
\hline $\begin{array}{c}\overline{C V a R}_{P, \Delta L} \\
\left(S D\left(C V a R_{P, \Delta L}\right)\right)\end{array}$ & $\begin{array}{c}\bar{r}_{P, \Delta L} \\
\left(S D\left(r_{P, \Delta L}\right)\right)\end{array}$ & $\begin{array}{c}\overline{C V a R}_{P, \Delta L} \\
\left(S D\left(C V a R_{P, \Delta L}\right)\right)\end{array}$ & $\begin{array}{c}\bar{r}_{P, \Delta L} \\
\left(S D\left(r_{P, \Delta L}\right)\right)\end{array}$ & & $\begin{array}{c}\overline{C V a R}_{P, \Delta L} \\
\left(\operatorname{SD}\left(\operatorname{CVaR}_{P, \Delta L}\right)\right)\end{array}$ & $\begin{array}{c}\bar{r}_{P, \Delta L} \\
\left(S D\left(r_{P, \Delta L}\right)\right)\end{array}$ \\
\hline \multirow{4}{*}{$\begin{array}{l}-0.04500 \\
(0.01396)\end{array}$} & \multirow{4}{*}{$\begin{array}{c}-0.03722 \\
(0.00997)\end{array}$} & \multirow{4}{*}{$\begin{array}{l}-0.76149 \\
(0.64170)\end{array}$} & \multirow{4}{*}{$\begin{array}{c}-0.11665 \\
(0.07573)\end{array}$} & 0.90 & $\begin{array}{c}-0.53915 \\
(0.15770)\end{array}$ & $\begin{array}{c}0.06086 \\
(0.01088)\end{array}$ \\
\hline & & & & 0.95 & $\begin{array}{c}-0.64736 \\
(0.16082)\end{array}$ & $\begin{array}{c}0.07179 \\
(0.01093)\end{array}$ \\
\hline & & & & 0.97 & $\begin{array}{c}-0.71960 \\
(0.16201)\end{array}$ & $\begin{array}{c}0.07904 \\
(0.01095)\end{array}$ \\
\hline & & & & 0.99 & $\begin{array}{c}-0.85924 \\
(0.16303)\end{array}$ & $\begin{array}{c}0.09303 \\
(0.01101)\end{array}$ \\
\hline
\end{tabular}

\begin{tabular}{|c|c|c|c|c|c|c|}
\hline \multicolumn{7}{|c|}{$\alpha_{\Delta L}=0.95$ and $V a R_{P, \Delta L}=-0.04685$} \\
\hline \multicolumn{2}{|c|}{ Deterministic equal weights } & \multicolumn{2}{|c|}{ Deterministic un-equal weights } & \multirow{2}{*}{$\begin{array}{l}\beta_{1} \\
= \\
\beta_{2}\end{array}$} & \multicolumn{2}{|c|}{ Probabilistic unequal weights } \\
\hline $\begin{array}{c}\overline{C V a R}_{P, \Delta L} \\
\left(S D\left(\operatorname{CVaR}_{P, \Delta L}\right)\right)\end{array}$ & $\begin{array}{c}\bar{r}_{P, \Delta L} \\
\left(S D\left(r_{P, \Delta L}\right)\right)\end{array}$ & $\begin{array}{c}\overline{C V a R}_{P, \Delta L} \\
\left(S D\left(C V a R_{P, \Delta L}\right)\right)\end{array}$ & $\begin{array}{c}\bar{r}_{P, \Delta L} \\
\left(S D\left(r_{P, \Delta L}\right)\right)\end{array}$ & & $\begin{array}{c}\overline{C V a R}_{P, \Delta L} \\
\left(S D\left(C V a R_{P, \Delta L}\right)\right)\end{array}$ & $\begin{array}{c}\bar{r}_{P, \Delta L} \\
\left(S D\left(r_{P, \Delta L}\right)\right)\end{array}$ \\
\hline \multirow{4}{*}{$\begin{array}{l}-0.04685 \\
(0.01484)\end{array}$} & \multirow{4}{*}{$\begin{array}{l}-0.03722 \\
(0.00997)\end{array}$} & \multirow{4}{*}{$\begin{array}{l}-1.44291 \\
(1.25322)\end{array}$} & \multirow{4}{*}{$\begin{array}{c}-0.11665 \\
(0.07573)\end{array}$} & 0.90 & $\begin{array}{c}-0.97007 \\
(0.30775)\end{array}$ & $\begin{array}{c}0.06316 \\
(0.00893)\end{array}$ \\
\hline & & & & 0.95 & $\begin{array}{c}-1.18925 \\
(0.31824)\end{array}$ & $\begin{array}{c}0.07387 \\
(0.00925)\end{array}$ \\
\hline & & & & 0.97 & $\begin{array}{c}-1.33570 \\
(0.32328)\end{array}$ & $\begin{array}{c}0.08096 \\
(0.00936)\end{array}$ \\
\hline & & & & 0.99 & $\begin{array}{c}-1.61915 \\
(0.32973) \\
\end{array}$ & $\begin{array}{c}0.09458 \\
(0.00970)\end{array}$ \\
\hline
\end{tabular}

\begin{tabular}{|c|c|c|c|c|c|c|}
\hline \multicolumn{7}{|c|}{$\alpha_{\Delta L}=0.97$ and $V a R_{P, \Delta L}=-0.04809$} \\
\hline \multicolumn{2}{|c|}{ Deterministic equal weights } & \multicolumn{2}{|c|}{ Deterministic un-equal weights } & \multirow{2}{*}{$\begin{array}{l}\beta_{1} \\
= \\
\beta_{2}\end{array}$} & \multicolumn{2}{|c|}{ Probabilistic unequal weights } \\
\hline $\begin{array}{c}\overline{C V a R}_{P, \Delta L} \\
\left(\operatorname{SD}\left(\operatorname{CVaR}_{P, \Delta L}\right)\right)\end{array}$ & $\begin{array}{c}\bar{r}_{P, \Delta L} \\
\left(S D\left(r_{P, \Delta L}\right)\right)\end{array}$ & $\begin{array}{c}\overline{C V a R}_{P, \Delta L} \\
\left(\operatorname{SD}\left(\mathrm{CVaR}_{P, \Delta L}\right)\right)\end{array}$ & $\begin{array}{c}\bar{r}_{P, \Delta L} \\
\left(S D\left(r_{P, \Delta L}\right)\right)\end{array}$ & & $\begin{array}{c}\overline{C V a R}_{P, \Delta L} \\
\left(\operatorname{SD}\left(\mathrm{CVaR}_{P, \Delta L}\right)\right)\end{array}$ & $\begin{array}{c}\bar{r}_{P, \Delta L} \\
\left(S D\left(r_{P, \Delta L}\right)\right)\end{array}$ \\
\hline \multirow{4}{*}{$\begin{array}{c}-0.04809 \\
(0.01549)\end{array}$} & \multirow{4}{*}{$\begin{array}{c}-0.03722 \\
(0.00997)\end{array}$} & \multirow{4}{*}{$\begin{array}{c}-2.33337 \\
(2.05814)\end{array}$} & \multirow{4}{*}{$\begin{array}{c}-0.11665 \\
(0.07573)\end{array}$} & 0.90 & $\begin{array}{l}-1.53796 \\
(0.52637)\end{array}$ & $\begin{array}{c}0.06394 \\
(0.00853)\end{array}$ \\
\hline & & & & 0.95 & $\begin{array}{l}-1.90098 \\
(0.54583)\end{array}$ & $\begin{array}{c}0.07477 \\
(0.00871)\end{array}$ \\
\hline & & & & 0.97 & $\begin{array}{c}-2.14391 \\
(0.55474)\end{array}$ & $\begin{array}{c}0.08191 \\
(0.00887)\end{array}$ \\
\hline & & & & 0.99 & $\begin{array}{l}-2.61445 \\
(0.56683)\end{array}$ & $\begin{array}{c}0.09561 \\
(0.00925)\end{array}$ \\
\hline
\end{tabular}




\begin{tabular}{|c|c|c|c|c|c|c|}
\hline \multicolumn{7}{|c|}{$\alpha_{\Delta L}=0.99$ and $V a R_{P, \Delta L}=-0.05062$} \\
\hline \multicolumn{2}{|c|}{ Deterministic equal weights } & \multicolumn{2}{|c|}{ Deterministic un-equal weights } & \multirow{2}{*}{$\begin{array}{c}\beta_{1} \\
= \\
\beta_{2}\end{array}$} & \multicolumn{2}{|c|}{ Probabilistic unequal weights } \\
\hline $\begin{array}{c}\overline{C V a R}_{P, \Delta L} \\
\left(\operatorname{SD}\left(\operatorname{CVaR}_{P, \Delta L}\right)\right)\end{array}$ & $\begin{array}{c}\bar{r}_{P, \Delta L} \\
\left(S D\left(r_{P, \Delta L}\right)\right)\end{array}$ & $\begin{array}{c}\overline{C V a R}_{P, \Delta L} \\
\left(\operatorname{SD}\left(\operatorname{CVaR}_{P, \Delta L}\right)\right)\end{array}$ & $\begin{array}{c}\bar{r}_{P, \Delta L} \\
\left(S D\left(r_{P, \Delta L}\right)\right)\end{array}$ & & $\begin{array}{c}\overline{C V a R}_{P, \Delta L} \\
\left(\operatorname{SD}\left(\operatorname{CVaR}_{P, \Delta L}\right)\right)\end{array}$ & $\begin{array}{c}\bar{r}_{P, \Delta L} \\
\left(S D\left(r_{P, \Delta L}\right)\right)\end{array}$ \\
\hline \multirow{4}{*}{$\begin{array}{c}-0.05062 \\
(0.01701)\end{array}$} & \multirow{4}{*}{$\begin{array}{l}-0.03722 \\
(0.00997)\end{array}$} & \multirow{4}{*}{$\begin{array}{l}-6.65321 \\
(5.99087)\end{array}$} & \multirow{4}{*}{$\begin{array}{c}-0.11665 \\
(0.07573)\end{array}$} & 0.90 & $\begin{array}{l}-4.27579 \\
(1.67393)\end{array}$ & $\begin{array}{c}0.06459 \\
(0.00831)\end{array}$ \\
\hline & & & & 0.95 & $\begin{array}{c}-5.35692 \\
(1.74918) \\
\end{array}$ & $\begin{array}{r}0.07545 \\
(0.00852) \\
\end{array}$ \\
\hline & & & & 0.97 & $\begin{array}{l}-6.08020 \\
(1.78640)\end{array}$ & $\begin{array}{c}0.08269 \\
(0.00864)\end{array}$ \\
\hline & & & & 0.99 & $\begin{array}{c}-7.48341 \\
(1.83691) \\
\end{array}$ & $\begin{array}{c}0.09649 \\
(0.00894) \\
\end{array}$ \\
\hline
\end{tabular}

Table 2. Average and Standard deviation values of $C V a R_{P, \Delta L}$ and $r_{P, \Delta L}$ for the outof-sample data set

\begin{tabular}{|c|c|c|c|c|c|c|}
\hline \multicolumn{7}{|c|}{$\alpha_{\Delta L}=0.90$ and $V a R_{P, \Delta L}=-0.04200$} \\
\hline \multicolumn{2}{|c|}{ Deterministic equal weights } & \multicolumn{2}{|c|}{ Deterministic un-equal weights } & \multirow{2}{*}{$\begin{array}{l}\beta_{1} \\
= \\
\beta_{2}\end{array}$} & \multicolumn{2}{|c|}{$\begin{array}{l}\text { Probabilistic unequal weights } \\
\text { s. }\end{array}$} \\
\hline $\begin{array}{c}\overline{\operatorname{CVaR}}_{P, \Delta L} \\
\left(\operatorname{SD}\left(\mathrm{CVaR}_{P, \Delta L}\right)\right)\end{array}$ & $\begin{array}{c}\bar{r}_{P, \Delta L} \\
\left(S D\left(r_{P, \Delta L}\right)\right)\end{array}$ & $\begin{array}{c}\overline{\operatorname{CVaR}}_{P, \Delta L} \\
\left(\operatorname{SD}\left(\mathrm{CVaR}_{P, \Delta L}\right)\right)\end{array}$ & $\begin{array}{c}\bar{r}_{P, \Delta L} \\
\left(S D\left(r_{P, \Delta L}\right)\right)\end{array}$ & & $\begin{array}{c}\overline{\operatorname{CVaR}}_{P, \Delta L} \\
\left(\operatorname{SD}\left(\mathrm{CVaR}_{P, \Delta L}\right)\right)\end{array}$ & $\begin{array}{c}\bar{r}_{P, \Delta L} \\
\left(S D\left(r_{P, \Delta L}\right)\right)\end{array}$ \\
\hline \multirow{4}{*}{$\begin{array}{l}-0.04200 \\
(0.00723)\end{array}$} & \multirow{4}{*}{$\begin{array}{c}-0.03699 \\
(0.00632)\end{array}$} & \multirow{4}{*}{$\begin{array}{c}-0.44222 \\
(0.18898)\end{array}$} & \multirow{4}{*}{$\begin{array}{c}-0.08202 \\
(0.02311)\end{array}$} & 0.90 & $\begin{array}{c}-1.24918 \\
(0.16932)\end{array}$ & $\begin{array}{c}0.10598 \\
(0.01728)\end{array}$ \\
\hline & & & & 0.95 & $\begin{array}{l}-1.44203 \\
(0.17312)\end{array}$ & $\begin{array}{c}0.12443 \\
(0.01775)\end{array}$ \\
\hline & & & & 0.97 & $\begin{array}{c}-1.56796 \\
(0.17363)\end{array}$ & $\begin{array}{c}0.13693 \\
(0.01780)\end{array}$ \\
\hline & & & & 0.99 & $\begin{array}{l}-1.81186 \\
(0.17632)\end{array}$ & $\begin{array}{c}0.16092 \\
(0.01805)\end{array}$ \\
\hline
\end{tabular}

\begin{tabular}{|c|c|c|c|c|c|c|}
\hline \multicolumn{7}{|c|}{$\alpha_{\Delta L}=0.95$ and $V a R_{P, \Delta L}=-0.04333$} \\
\hline \multicolumn{2}{|c|}{ Deterministic equal weights } & \multicolumn{2}{|c|}{ Deterministic un-equal weights } & \multirow{2}{*}{$\begin{array}{c}\beta_{1} \\
= \\
\beta_{2}\end{array}$} & \multicolumn{2}{|c|}{ Probabilistic unequal weights } \\
\hline $\begin{array}{c}\overline{C V a R}_{P, \Delta L} \\
\left(\operatorname{SD}\left(\mathrm{CVaR}_{P, \Delta L}\right)\right)\end{array}$ & $\begin{array}{c}\bar{r}_{P, \Delta L} \\
\left(S D\left(r_{P, \Delta L}\right)\right)\end{array}$ & $\begin{array}{c}\overline{\operatorname{CVaR}}_{P, \Delta L} \\
\left(\operatorname{SD}\left(\mathrm{CVaR}_{P, \Delta L}\right)\right)\end{array}$ & $\begin{array}{c}\bar{r}_{P, \Delta L} \\
\left(S D\left(r_{P, \Delta L}\right)\right)\end{array}$ & & $\begin{array}{c}\overline{\operatorname{CVaR}}_{P, \Delta L} \\
\left(\operatorname{SD}\left(\mathrm{CVaR}_{P, \Delta L}\right)\right)\end{array}$ & $\begin{array}{c}\bar{r}_{P, \Delta L} \\
\left(S D\left(r_{P, \Delta L}\right)\right)\end{array}$ \\
\hline \multirow{4}{*}{$\begin{array}{c}-0.04333 \\
(0.00750)\end{array}$} & \multirow{4}{*}{$\begin{array}{c}-0.03699 \\
(0.00632)\end{array}$} & \multirow{4}{*}{$\begin{array}{c}-0.81715 \\
(0.36800)\end{array}$} & \multirow{4}{*}{$\begin{array}{c}-0.08202 \\
(0.02311)\end{array}$} & 0.90 & $\begin{array}{c}-2.29889 \\
(0.23820)\end{array}$ & $\begin{array}{c}0.11555 \\
(0.01296)\end{array}$ \\
\hline & & & & 0.95 & $\begin{array}{l}-2.68825 \\
(0.24460)\end{array}$ & $\begin{array}{c}0.13377 \\
(0.01339) \\
\end{array}$ \\
\hline & & & & 0.97 & $\begin{array}{c}-2.94827 \\
(0.25088)\end{array}$ & $\begin{array}{c}0.14567 \\
(0.01369)\end{array}$ \\
\hline & & & & 0.99 & $\begin{array}{l}-3.44317 \\
(0.25882)\end{array}$ & $\begin{array}{c}0.16925 \\
(0.01428)\end{array}$ \\
\hline
\end{tabular}




\begin{tabular}{|c|c|c|c|c|c|c|}
\hline \multicolumn{7}{|c|}{$\alpha_{\Delta L}=0.97$ and $V a R_{P, \Delta L}=-0.04421$} \\
\hline \multicolumn{2}{|c|}{ Deterministic equal weights } & \multicolumn{2}{|c|}{ Deterministic un-equal weights } & \multirow{2}{*}{$\begin{array}{l}\beta_{1} \\
= \\
\beta_{2}\end{array}$} & \multicolumn{2}{|c|}{$\begin{array}{l}\text { Probabilistic unequal weights } \\
\end{array}$} \\
\hline $\begin{array}{c}\overline{C V a R}_{P, \Delta L} \\
\left(S D\left(C V a R_{P, \Delta L}\right)\right)\end{array}$ & $\begin{array}{c}\bar{r}_{P, \Delta L} \\
\left(S D\left(r_{P, \Delta L}\right)\right)\end{array}$ & $\begin{array}{c}\overline{C V a R}_{P, \Delta L} \\
\left(\operatorname{SD}\left(\operatorname{CVaR}_{P, \Delta L}\right)\right)\end{array}$ & $\begin{array}{c}\bar{r}_{P, \Delta L} \\
\left(S D\left(r_{P, \Delta L}\right)\right)\end{array}$ & & $\begin{array}{c}\overline{C V a R}_{P, \Delta L} \\
\left(\operatorname{SD}\left(\mathrm{CVaR}_{P, \Delta L}\right)\right)\end{array}$ & $\begin{array}{c}\bar{r}_{P, \Delta L} \\
\left(S D\left(r_{P, \Delta L}\right)\right)\end{array}$ \\
\hline \multirow{4}{*}{$\begin{array}{c}-0.04421 \\
(0.00768)\end{array}$} & \multirow{4}{*}{$\begin{array}{c}-0.03699 \\
(0.00632)\end{array}$} & \multirow{4}{*}{$\begin{array}{c}-1.30456 \\
(0.60348)\end{array}$} & \multirow{4}{*}{$\begin{array}{c}-0.08202 \\
(0.02311)\end{array}$} & 0.90 & $\begin{array}{l}-3.72984 \\
(0.37080)\end{array}$ & $\begin{array}{c}0.11926 \\
(0.01283)\end{array}$ \\
\hline & & & & 0.95 & $\begin{array}{l}-4.56204 \\
(0.34077)\end{array}$ & $\begin{array}{c}0.12925 \\
(0.00967)\end{array}$ \\
\hline & & & & 0.97 & $\begin{array}{l}-4.81494 \\
(0.03938)\end{array}$ & $\begin{array}{c}0.14913 \\
(0.01341)\end{array}$ \\
\hline & & & & 0.99 & $\begin{array}{l}-5.64270 \\
(0.40335)\end{array}$ & $\begin{array}{c}0.17253 \\
(0.01383) \\
\end{array}$ \\
\hline
\end{tabular}

\begin{tabular}{|c|c|c|c|c|c|c|}
\hline \multicolumn{7}{|c|}{$\alpha_{\Delta L}=0.99$ and $V a R_{P, \Delta L}=-0.04590$} \\
\hline \multicolumn{2}{|c|}{ Deterministic equal weights } & \multicolumn{2}{|c|}{ Deterministic un-equal weights } & \multirow{2}{*}{$\begin{array}{l}\beta_{1} \\
= \\
\beta_{2}\end{array}$} & \multicolumn{2}{|c|}{ Probabilistic unequal weights } \\
\hline $\begin{array}{c}\overline{C V a R}_{P, \Delta L} \\
\left(\operatorname{SD}\left(\operatorname{CVaR}_{P, \Delta L}\right)\right)\end{array}$ & $\begin{array}{c}\bar{r}_{P, \Delta L} \\
\left(S D\left(r_{P, \Delta L}\right)\right)\end{array}$ & $\begin{array}{c}\overline{C V a R}_{P, \Delta L} \\
\left(\operatorname{SD}\left(\operatorname{CVaR}_{P, \Delta L}\right)\right)\end{array}$ & $\begin{array}{c}\bar{r}_{P, \Delta L} \\
\left(S D\left(r_{P, \Delta L}\right)\right)\end{array}$ & & $\begin{array}{c}\overline{C V a R}_{P, \Delta L} \\
\left(\operatorname{SD}\left(\mathrm{CVaR}_{P, \Delta L}\right)\right)\end{array}$ & $\begin{array}{c}\bar{r}_{P, \Delta L} \\
\left(S D\left(r_{P, \Delta L}\right)\right)\end{array}$ \\
\hline \multirow{4}{*}{$\begin{array}{c}-0.04590 \\
(0.00807)\end{array}$} & \multirow{4}{*}{$\begin{array}{c}-0.03699 \\
(0.00632)\end{array}$} & \multirow{4}{*}{$\begin{array}{c}-3.65777 \\
(1.75765)\end{array}$} & \multirow{4}{*}{$\begin{array}{c}-0.08202 \\
(0.02311)\end{array}$} & 0.90 & $\begin{array}{l}-10.86932 \\
(1.11025)\end{array}$ & $\begin{array}{c}0.12284 \\
(0.01317)\end{array}$ \\
\hline & & & & 0.95 & $\begin{array}{c}-12.82539 \\
(1.13895)\end{array}$ & $\begin{array}{c}0.14090 \\
(0.01357)\end{array}$ \\
\hline & & & & 0.97 & $\begin{array}{l}-14.11982 \\
(1.15804)\end{array}$ & $\begin{array}{c}0.15290 \\
(0.01386)\end{array}$ \\
\hline & & & & 0.99 & $\begin{array}{l}-16.60636 \\
(1.19280)\end{array}$ & $\begin{array}{c}0.1762) \\
(0.01438)\end{array}$ \\
\hline
\end{tabular}

Table 3. Percentage change in $\overline{C V a R}_{P, \Delta L}$ and $\bar{r}_{P, \Delta L}$ with respect to change in $\alpha_{\Delta L}$ and $\beta_{1}=\beta_{2}$ for both in-sample and out-of-sample data sets

\begin{tabular}{|c|c|c|c|c|c|c|c|}
\hline \multicolumn{8}{|c|}{ In-sample data set } \\
\hline & & $\begin{array}{c}\% \Delta \overline{C V a R}_{P, \Delta L} \\
\text { deterministic } \\
\text { equal } \\
\text { weights } \\
\end{array}$ & $\begin{array}{c}\% \Delta \overline{C V a R}_{P, \Delta L} \\
\text { deterministic } \\
\text { un-equal } \\
\text { weights } \\
\end{array}$ & $\begin{array}{c}\% \Delta \overline{C V a R}_{P, \Delta L} \\
\text { probabilistic } \\
\text { case }\end{array}$ & $\begin{array}{c}\% \Delta \bar{r}_{P, \Delta L} \\
\text { deterministi } \\
\text { c equal } \\
\text { weights } \\
\end{array}$ & $\begin{array}{c}\% \Delta \bar{r}_{P, \Delta L} \\
\text { deterministi } \\
\text { c un-equal } \\
\text { weights }\end{array}$ & $\begin{array}{c}\% \Delta \bar{r}_{P, \Delta L} \\
\text { probabil } \\
\text { istic } \\
\text { case } \\
\end{array}$ \\
\hline \multirow[t]{3}{*}{$\Delta \alpha_{\Delta L}$} & $90 \%$ to $95 \%$ & $4.11 \%$ & $89.49 \%$ & - & $0 \%$ & $0 \%$ & - \\
\hline & $95 \%$ to $97 \%$ & $2.65 \%$ & $61.72 \%$ & - & $0 \%$ & $0 \%$ & - \\
\hline & $97 \%$ to $99 \%$ & $5.26 \%$ & $185.13 \%$ & - & $0 \%$ & $0 \%$ & - \\
\hline \multirow[t]{7}{*}{$\begin{array}{l}\Delta \beta_{1}, \Delta \beta_{2} \\
\text { and } \alpha_{\Delta L}\end{array}$} & $\begin{array}{l}90 \% \text { to } 95 \% \\
\text { and } 90 \%\end{array}$ & - & - & $20.07 \%$ & - & - & $17.96 \%$ \\
\hline & $\begin{array}{l}90 \% \text { to } 95 \% \\
\text { and } 95 \%\end{array}$ & - & - & $22.59 \%$ & - & - & $16.96 \%$ \\
\hline & $\begin{array}{l}90 \% \text { to } 95 \% \\
\text { and } 97 \%\end{array}$ & - & - & $23.61 \%$ & - & - & $16.94 \%$ \\
\hline & $\begin{array}{l}90 \% \text { to } 95 \% \\
\text { and } 99 \%\end{array}$ & - & - & $25.29 \%$ & - & - & $16.81 \%$ \\
\hline & $\begin{array}{l}95 \% \text { to } 97 \% \\
\text { and } 90 \%\end{array}$ & - & - & $11.16 \%$ & - & - & $10.10 \%$ \\
\hline & $\begin{array}{l}95 \% \text { to } 97 \% \\
\text { and } 95 \%\end{array}$ & - & - & $12.31 \%$ & - & - & $9.60 \%$ \\
\hline & $95 \%$ to $97 \%$ & - & - & $12.78 \%$ & - & - & $9.55 \%$ \\
\hline
\end{tabular}




\begin{tabular}{|l|l|c|c|c|c|c|c|}
\hline & & & & & & \\
\hline $\begin{array}{l}\text { and 97\% } \\
\begin{array}{l}95 \% \text { to } 97 \% \\
\text { and 99\% }\end{array}\end{array}$ & - & - & $13.51 \%$ & - & - & $9.60 \%$ \\
\hline $\begin{array}{l}97 \% \text { to 99\% } \\
\text { and 90\% }\end{array}$ & - & - & $19.40 \%$ & - & - & $17.70 \%$ \\
\hline $\begin{array}{l}97 \% \text { to 99\% } \\
\text { and 95\% }\end{array}$ & - & - & $21.22 \%$ & - & - & $16.82 \%$ \\
\hline $\begin{array}{l}97 \% \text { to 99\% } \\
\text { and 97\% }\end{array}$ & - & - & $21.95 \%$ & - & - & $16.73 \%$ \\
\hline $\begin{array}{l}97 \% \text { to 99\% } \\
\text { and 99\% }\end{array}$ & - & - & $23.08 \%$ & - & - & $16.69 \%$ \\
\hline
\end{tabular}

\begin{tabular}{|c|c|c|c|c|c|c|c|}
\hline \multicolumn{8}{|c|}{ Out-of-sample data set } \\
\hline & & $\begin{array}{c}\mathrm{c}_{0 \Delta \overline{C V a R}_{P, \Delta L}} \\
\text { deterministi } \\
\text { c equal } \\
\text { weights }\end{array}$ & $\begin{array}{c}{ }_{\% \Delta \Delta \overline{C V a R}_{P, \Delta L}} \\
\text { deterministi } \\
\text { c un-equal } \\
\text { weights }\end{array}$ & $\begin{array}{c}\% \Delta \overline{C V a R}_{P, \Delta L} \\
\text { probabilistic } \\
\text { case }\end{array}$ & $\begin{array}{c}\% \Delta \bar{r}_{P, \Delta L} \\
\text { deterministi } \\
\text { c equal } \\
\text { weights }\end{array}$ & $\begin{array}{c}\% \Delta \bar{r}_{P, \Delta L} \\
\text { deterministi } \\
\text { c un-equal } \\
\text { weights }\end{array}$ & $\begin{array}{c}\% \Delta \bar{r}_{P, \Delta L} \\
\text { probabil } \\
\text { istic } \\
\text { case }\end{array}$ \\
\hline \multirow[t]{3}{*}{$\Delta \alpha_{\Delta L}$} & $90 \%$ to $95 \%$ & $3.17 \%$ & $84.79 \%$ & - & $0 \%$ & $0 \%$ & - \\
\hline & $95 \%$ to $97 \%$ & $2.03 \%$ & $59.65 \%$ & - & $0 \%$ & $0 \%$ & - \\
\hline & $97 \%$ to $99 \%$ & $3.82 \%$ & $180.38 \%$ & - & $0 \%$ & $0 \%$ & - \\
\hline \multirow[t]{12}{*}{$\begin{array}{l}\Delta \beta_{1}, \Delta \beta_{2} \\
\text { and } \alpha_{\Delta L}\end{array}$} & $\begin{array}{l}90 \% \text { to } 95 \% \\
\text { and } 90 \%\end{array}$ & - & - & $15.44 \%$ & - & - & $17.41 \%$ \\
\hline & $\begin{array}{l}90 \% \text { to } 95 \% \\
\text { and } 95 \%\end{array}$ & - & - & $16.94 \%$ & - & - & $15.77 \%$ \\
\hline & $\begin{array}{l}90 \% \text { to } 95 \% \\
\text { and } 97 \%\end{array}$ & - & - & $22.32 \%$ & - & - & $8.38 \%$ \\
\hline & $\begin{array}{l}90 \% \text { to } 95 \% \\
\text { and } 99 \%\end{array}$ & - & - & $18.00 \%$ & - & - & $14.70 \%$ \\
\hline & $\begin{array}{l}95 \% \text { to } 97 \% \\
\text { and } 90 \%\end{array}$ & - & - & $8.74 \%$ & - & - & $10.05 \%$ \\
\hline & $\begin{array}{l}95 \% \text { to } 97 \% \\
\text { and } 95 \%\end{array}$ & - & - & $9.67 \%$ & - & - & $8.90 \%$ \\
\hline & $\begin{array}{l}95 \% \text { to } 97 \% \\
\text { and } 97 \%\end{array}$ & - & - & $5.55 \%$ & - & - & $15.38 \%$ \\
\hline & $\begin{array}{l}95 \% \text { to } 97 \% \\
\text { and } 99 \%\end{array}$ & - & - & $10.10 \%$ & - & - & $8.52 \%$ \\
\hline & $\begin{array}{l}97 \% \text { to } 99 \% \\
\text { and } 90 \%\end{array}$ & - & - & $15.55 \%$ & - & - & $17.52 \%$ \\
\hline & $\begin{array}{l}97 \% \text { to } 99 \% \\
\text { and } 95 \%\end{array}$ & - & - & $16.79 \%$ & - & - & $16.19 \%$ \\
\hline & $\begin{array}{l}97 \% \text { to } 99 \% \\
\text { and } 97 \%\end{array}$ & - & - & $17.19 \%$ & - & - & $15.69 \%$ \\
\hline & $\begin{array}{l}97 \% \text { to } 99 \% \\
\text { and } 99 \%\end{array}$ & - & - & $17.61 \%$ & - & - & $15.29 \%$ \\
\hline
\end{tabular}

Utilizing tables $1 \& 2$ it is evident that for an increase in $\alpha_{\Delta L}$ values, the corresponding increase in $\overline{C V a R}_{P, \Delta L}$ or $\bar{r}_{P, \Delta L}$, for the deterministic equal weights, deterministic unequal weights and the probabilistic case are of different percentages and convey separate findings. The summary of this is shown in table 3. What is interesting from these readings is the fact that for any particular value of $\beta_{1}=\beta_{2}$ and $\alpha_{\Delta L}$ or $\Delta \alpha_{\Delta L}$ as the case may be, the variations in percentage values of $\overline{C V a R}_{P, \Delta L}$ or $\bar{r}_{P, \Delta L}$ when one compares the results of in- 
sample to that of out-of-sample output is on an average in the range of $-24.46 \%$ and $-3.97 \%$ respectively. Added to that, the $S D\left(C V a R_{P, \Delta L}\right)$ and $S D\left(r_{P, \Delta L}\right)$ values are also very low. Hence one may reasonable conclude that our probabilistic optimization model is very robust as it should be. The next interesting observation is the fact that for both deterministic equal weights and deterministic unequal weights case, the increase in $\bar{r}_{P, \Delta L}$ is zero, in-spite of the fact that $\alpha_{\Delta L}$ hence, both $\operatorname{VaR}_{P, \Delta L}$ as well as $\overline{C V a R}_{P, \Delta L}$ values increase. This may be attributed to the fact that one of the main input parameters, $r_{i, \Delta L}, i=1, \ldots 12$, is considered deterministic. While for the non-deterministic case we obtain the results as predicted, as it concurs with the general notion that an increase in the return values of the portfolio has an equally proportionate compensating increase in the risk values.

We run our model for different values $\alpha_{\Delta L}$ and $\beta_{1}=\beta_{2}$, which result in 48 different combinations of runs which are shown in figures 1 to 6 . The outputs are generic in nature. Hence rather than give a detailed explanation for each of the output separately we highlight the important observations and give the analysis for the same.

First let us consider figure 1(a) which shows the 3D scatter plots of $C V a R_{P, \Delta L}, r_{P, \Delta L}$ and $\operatorname{VaR}_{P, \Delta L}$ for 2497 number of simulated runs considering each of the combinations pertaining to (i) $\alpha_{\Delta L}=0.90, \beta_{1}=\beta_{2}=0.90$, depicted by circular (o) plots; (ii) $\alpha_{\Delta L}=$ $0.90, \beta_{1}=\beta_{2}=0.95$, shown by star (*) plots; (iii) $\alpha_{\Delta L}=0.90, \beta_{1}=\beta_{2}=0.97$, illustrated using plus ( + ) plots, and finally (iii) $\alpha_{\Delta L}=0.90, \beta_{1}=\beta_{2}=0.99$, highlighted utilizing square ( $\square$ ) plots. From figure $1(\mathrm{a})$ it is evident that as the values of $\beta_{1}$ and $\beta_{2}$ increase/decrease the values of $C V a R_{P, \Delta L}$ also increase/decrease. This means that as we become more/less reliable with respect to the estimated values of the input data, the conditional value at risk values also increase/decrease proportionally, but in the negative direction. This is logical as we consider the return distribution to be that of loss only.

The next interesting point is the way the four set of scatter plots (considering four different combinations of $\beta_{1}, \beta_{2}$ ) are spread with respect to the value at risk. From our model it is clear, that $\operatorname{VaR}_{P, \Delta L}$, values are same for all the four different combinations of $\alpha_{\Delta L}, \beta_{1}$ and $\beta_{2}$. This is true as the simulated values of $V a R_{P, \Delta L}$ depend only on, $\alpha_{\Delta L}$, irrespective of the reliability levels. The third observation worth highlighting is the general trend of the return values with respect to the change of $\beta_{1}, \beta_{2}$. It is noticed that with an increase/decrease in the reliability levels, the shift in the trend of the return values of the portfolio signify that we move away/towards the portfolio's average value. This means that one is less/more certain about the average returns of the portfolio. A similar set of conclusions can be deduced if one now concentrates on figure 1(b) which pertains to the four different sets of scatter plots, but now for the out-of-sample data series. The only change in figure 1(b) with respect to figure 1(a) is the greater spread of the value at risk values for out-of-sample data series than that for the in-sample data points. 


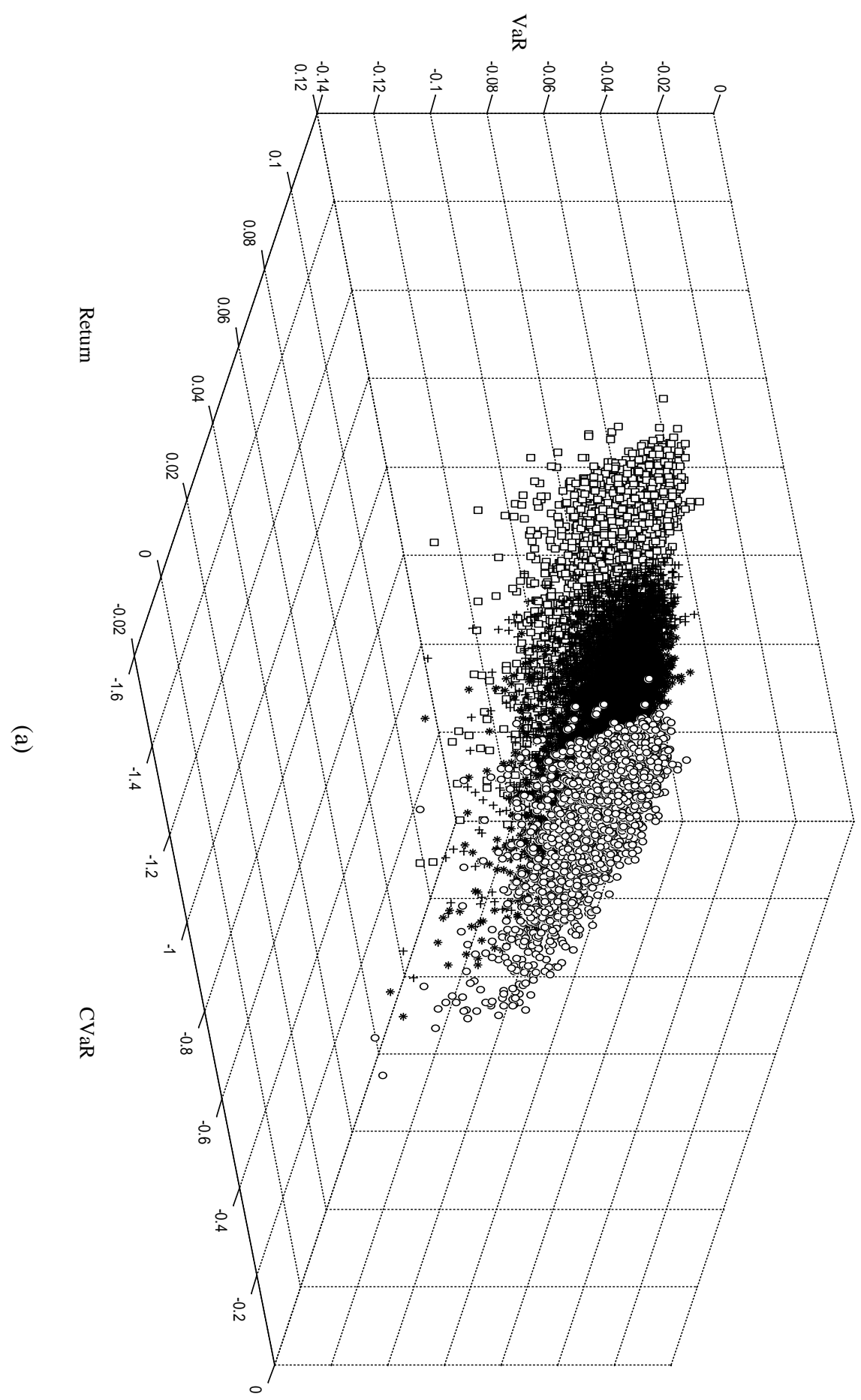



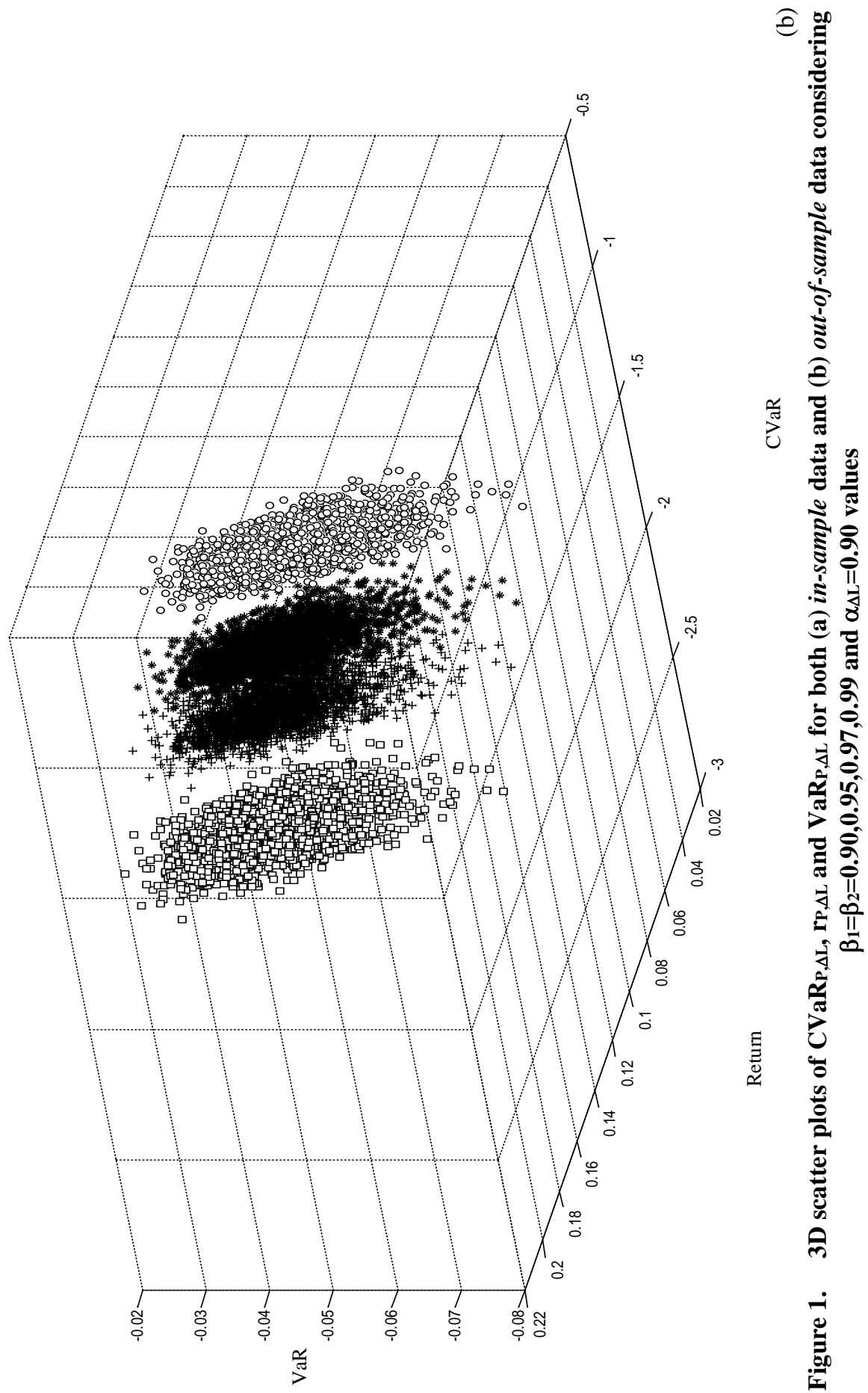


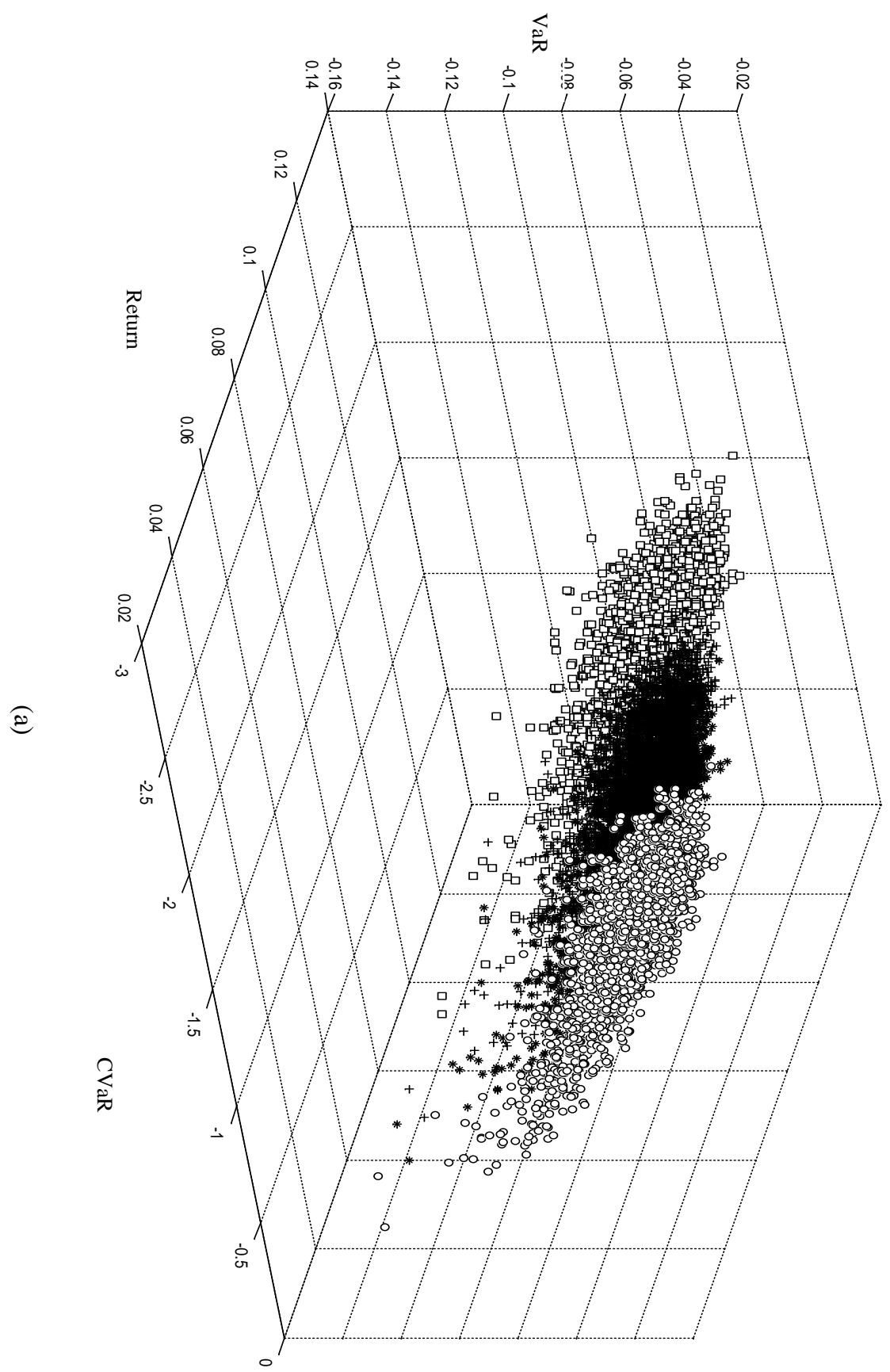



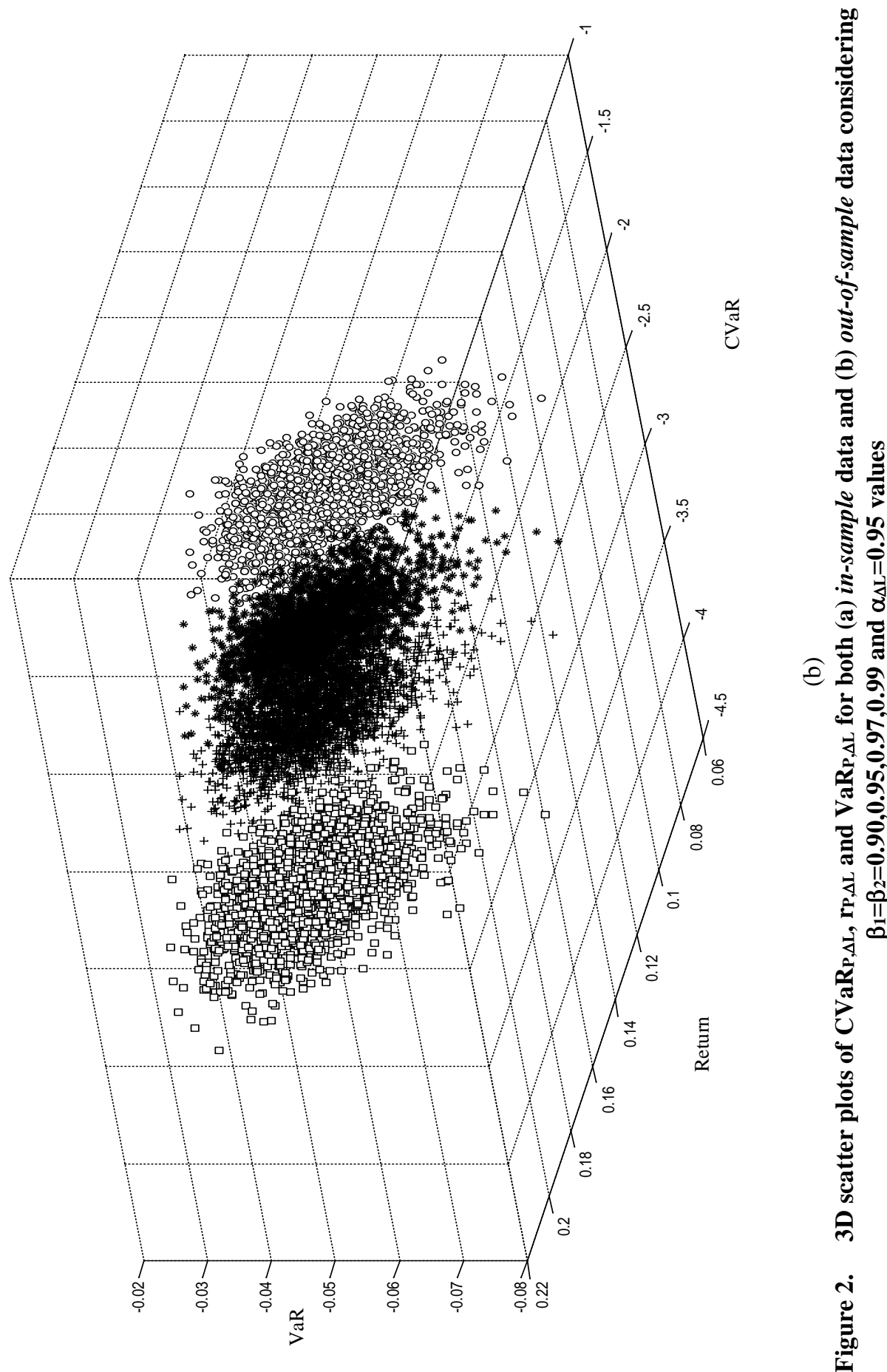


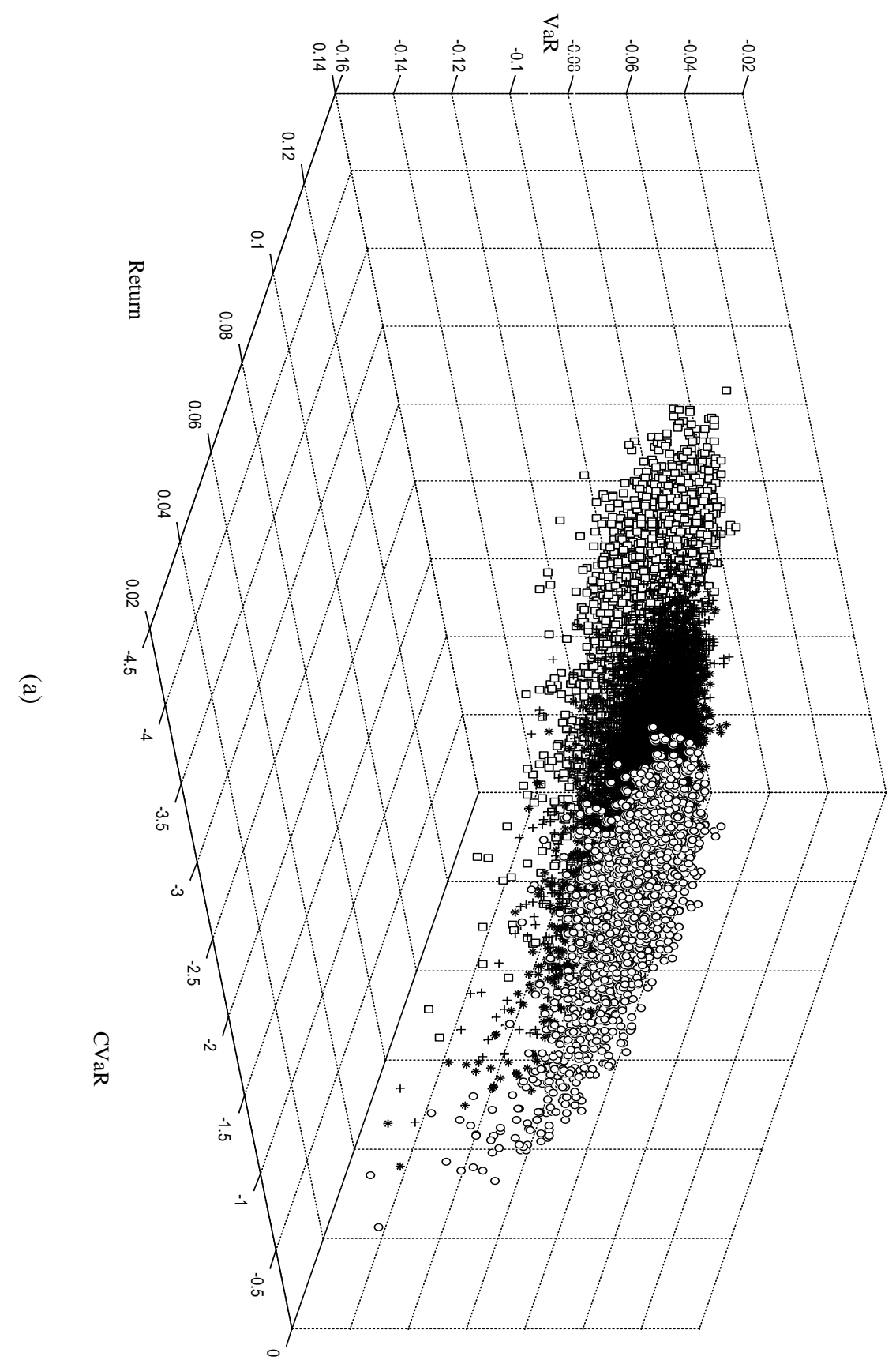




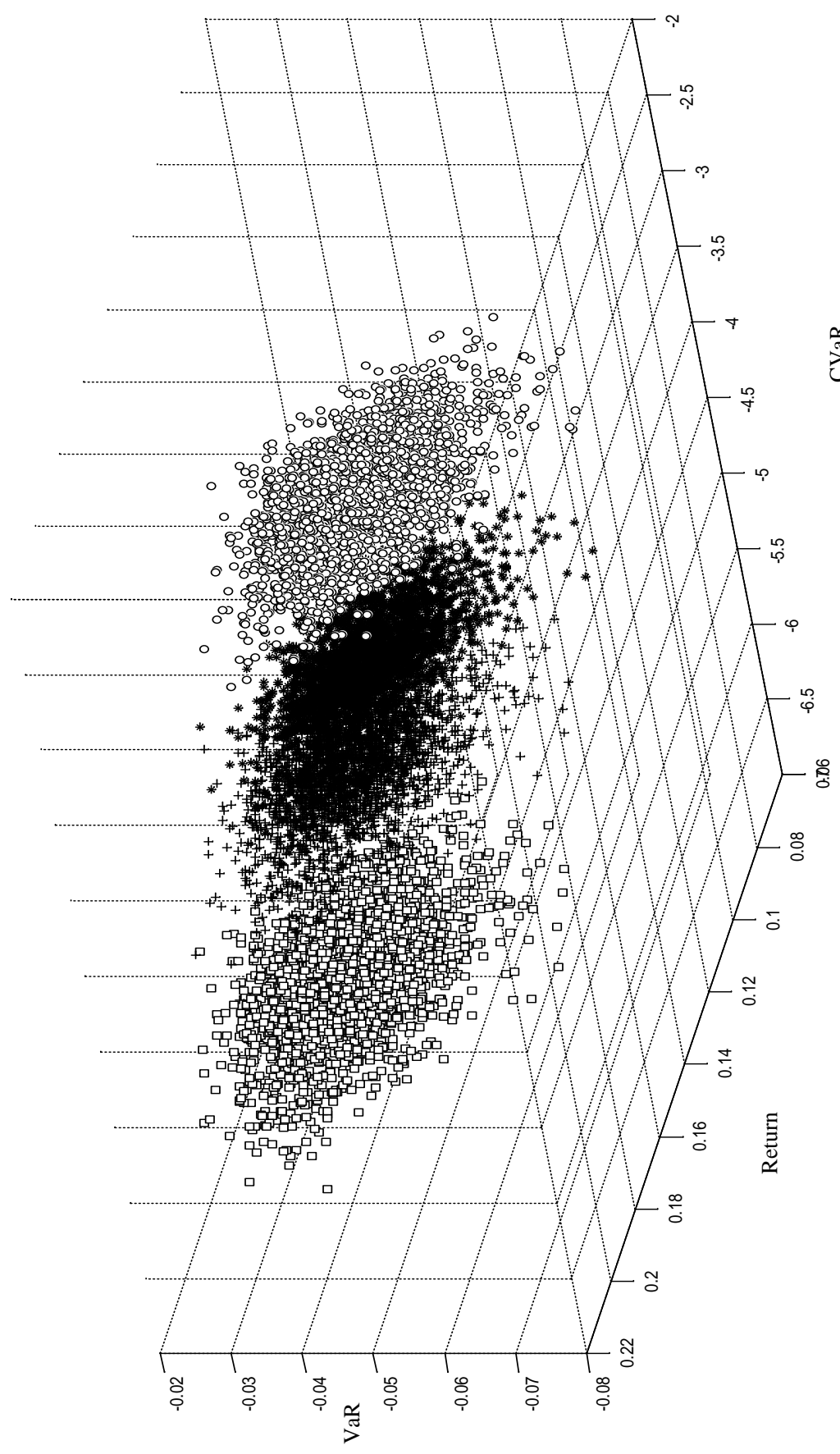

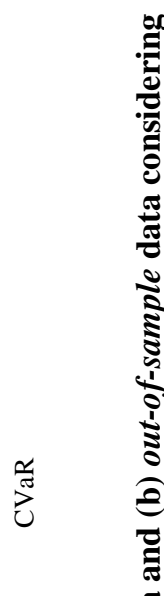

흔

ถั้

ड़

เ

อิ ㅎํำ

옹

ये

ํํำ

$>$

ํํํ

ये

औे

गे

فำ 


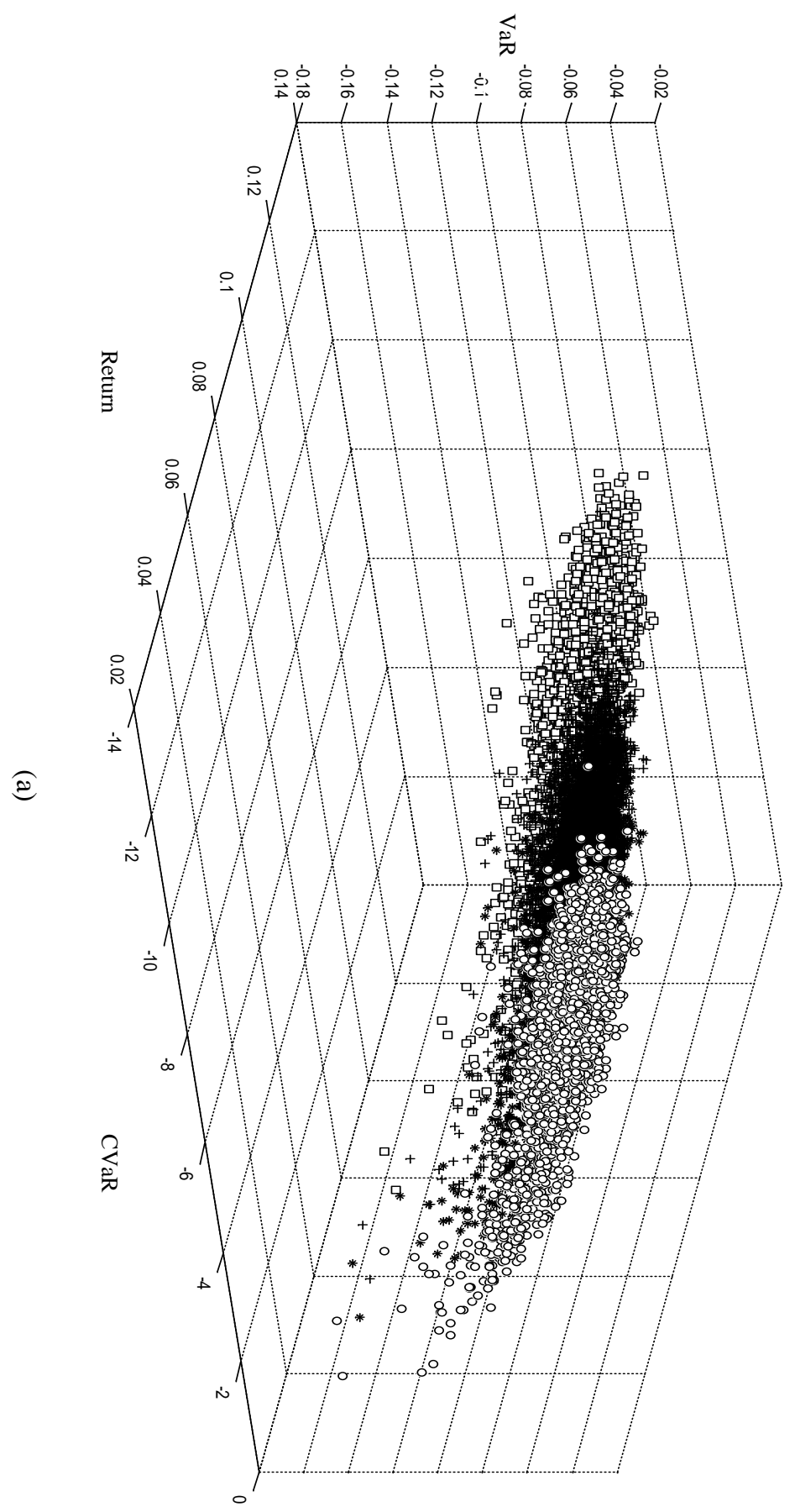



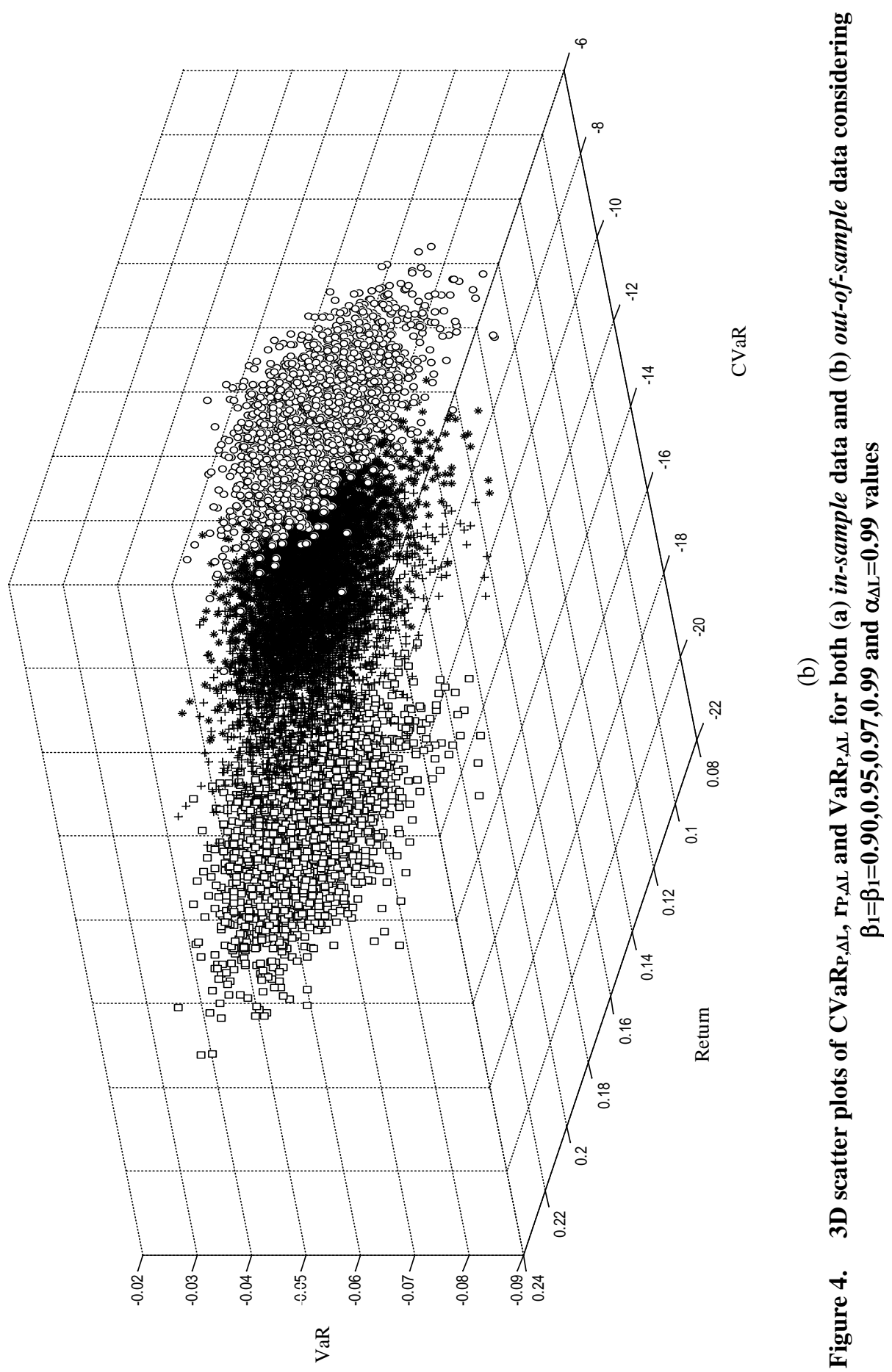


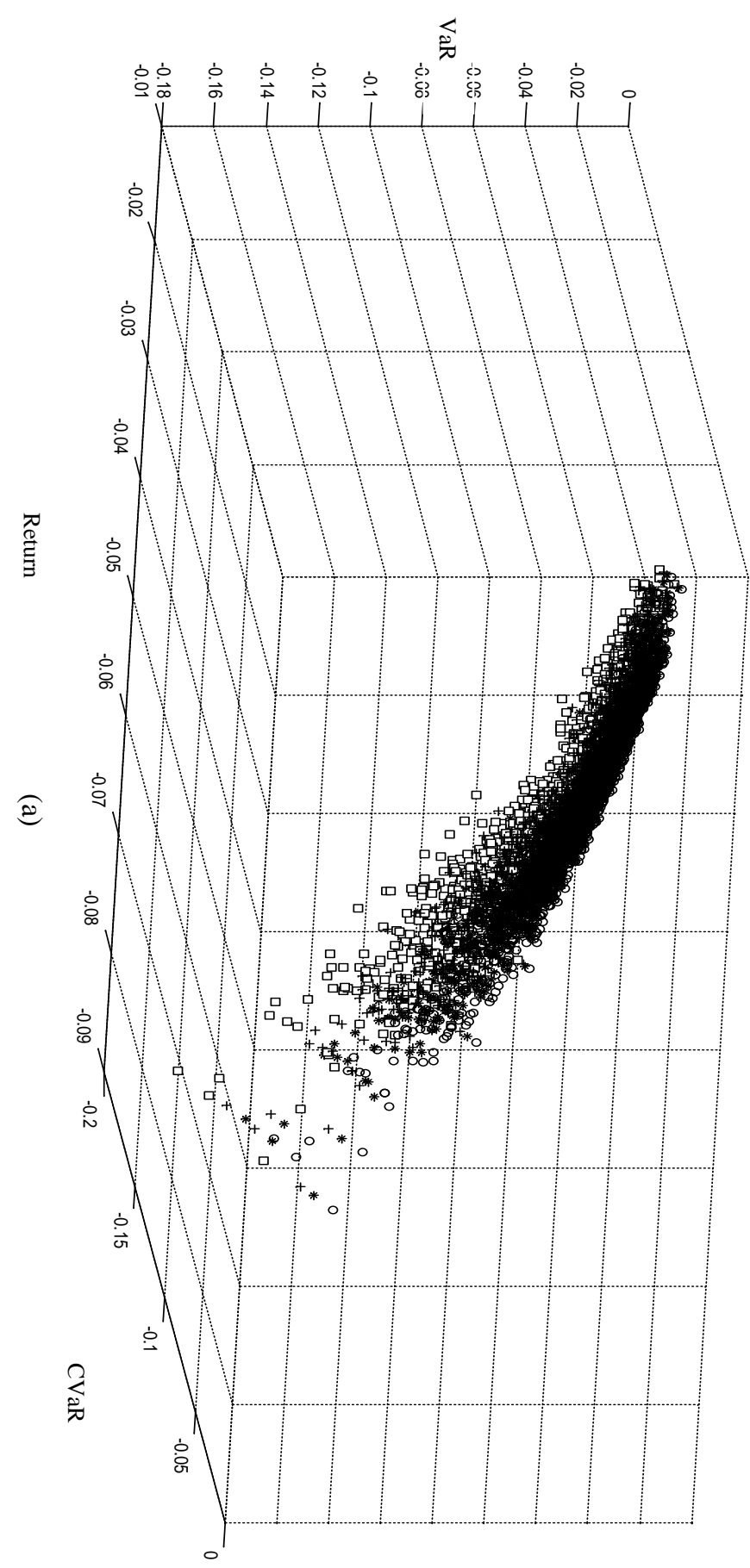




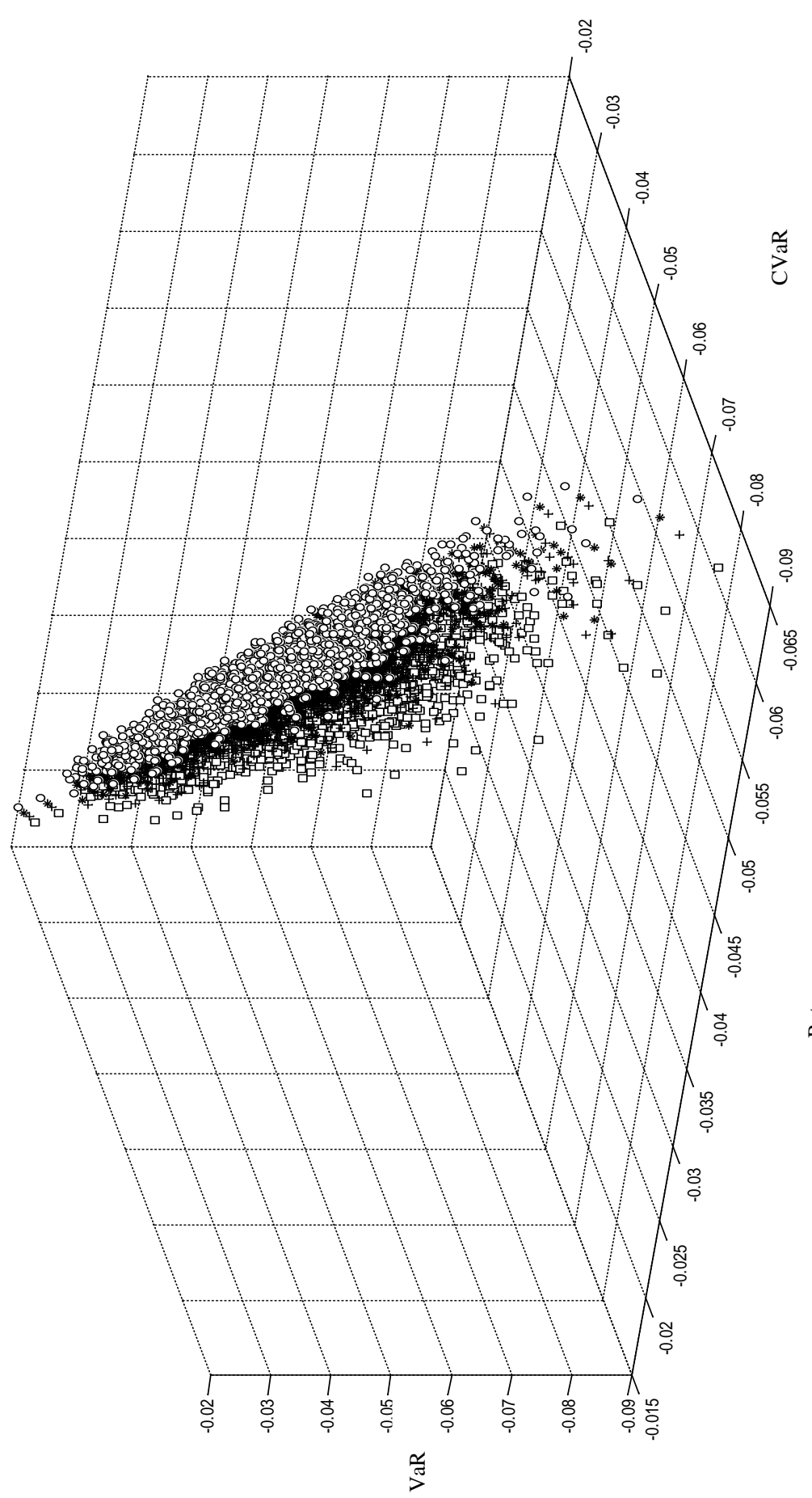

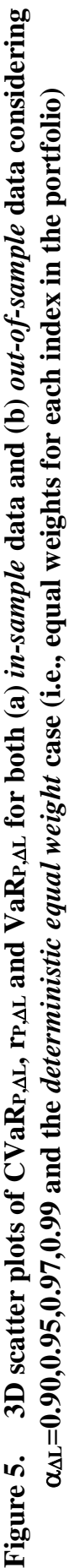




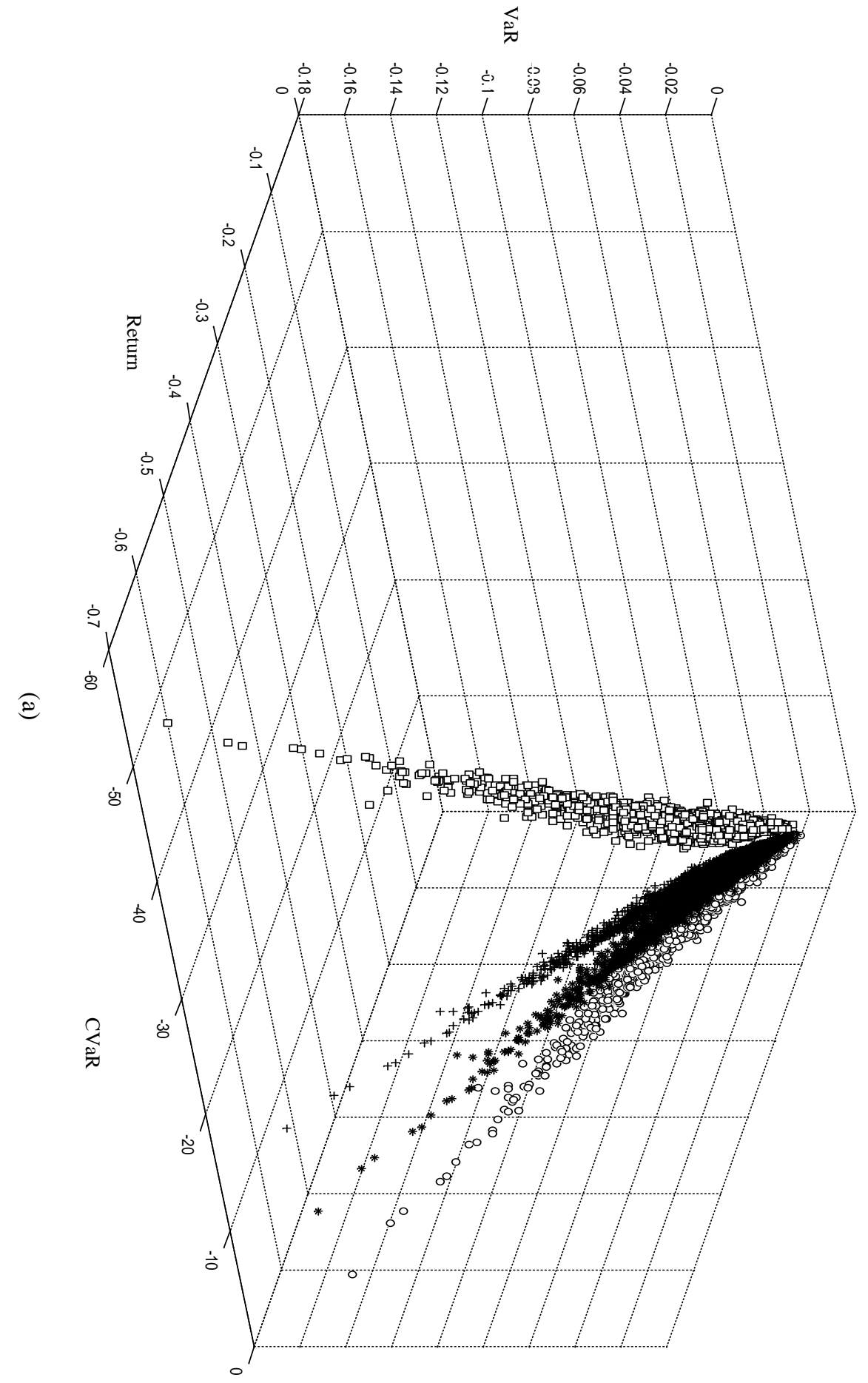



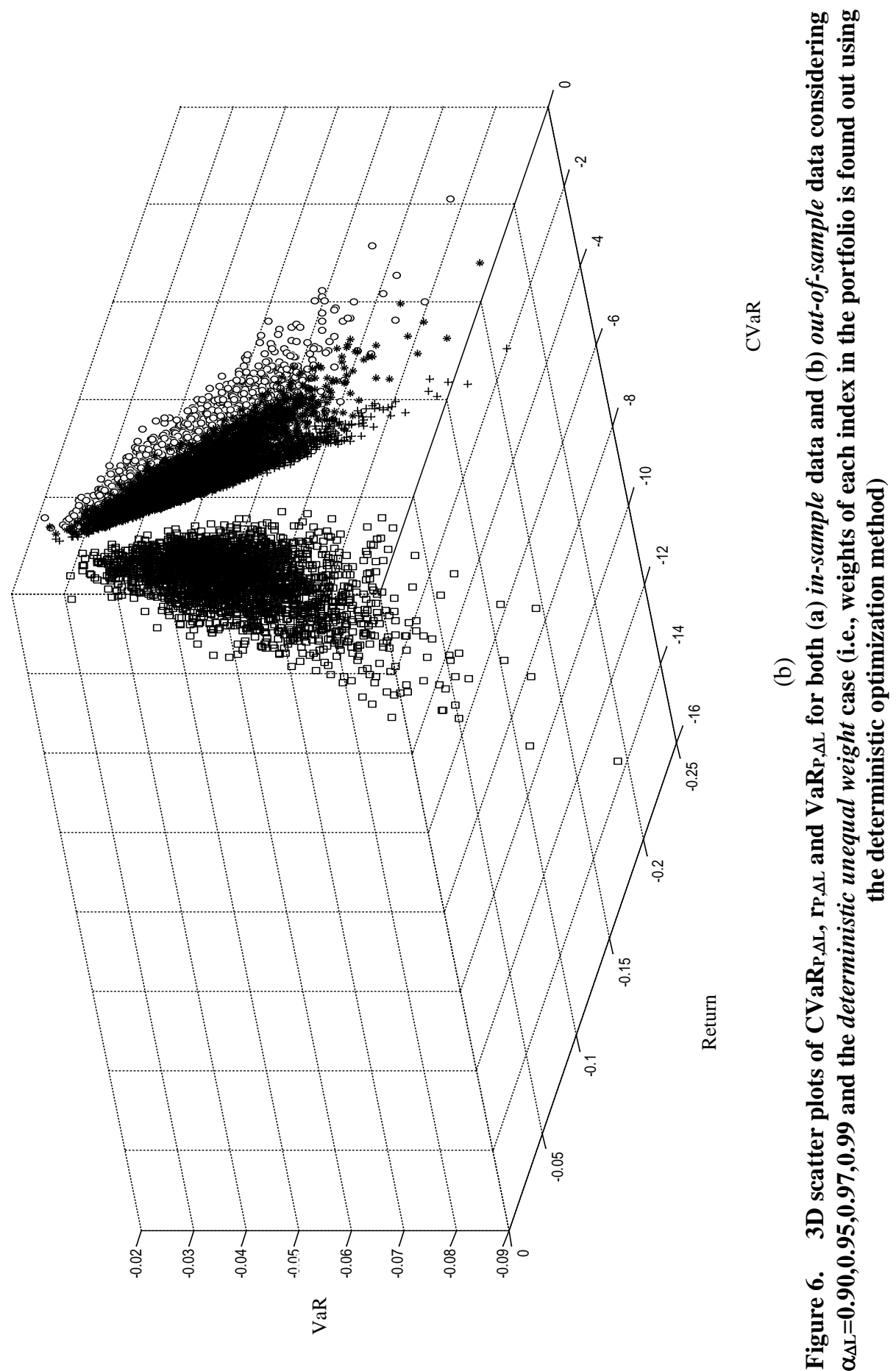
On a similar line one can deduce analogous set of conclusions from figures 2, figures 3 and figures 4 which relate to the scatter plots corresponding to values of $\alpha_{\Delta L}=95 \%, 97 \%$ and $99 \%$ respectively.

The next set of figures, i.e., figures 5 and 6 depict the 3D scatter plots of $C V a R_{P, \Delta L}, r_{P, \Delta L}$ and $\operatorname{VaR}_{P, \Delta L}$ for the deterministic equal weight and the deterministic unequal weight examples respectively. Figure 5(a) shows the plot for the in-sample data set, while the outof-sample case is shown in figure 5(b). In both these figures it is worth noting that a change in the $\alpha_{P, \Delta L}$ value does change the $\operatorname{VaR}_{P, \Delta L}$ values which is predictable, but what is interesting is the general trend of the scatter plot envelopes formed for the four different values of $\alpha_{P, \Delta L}$. The reader can easily make out that they form distinct contours which are almost parallel non-overlapping envelopes. The envelope contour formed for $\alpha_{P, \Delta L}=90 \%$ is the outer most one which encompasses all the other three envelopes contours such that the scatter plot for $\alpha_{P, \Delta L}=99 \%$ is at the lowest level followed by $\alpha_{P, \Delta L}=97 \%$ and then by $\alpha_{P, \Delta L}=95 \%$ respectively. Thus these contours depict the fact that a change in $\alpha_{P, \Delta L}$ only changes $C V a R_{P, \Delta L}$ and not $r_{P, \Delta L}$ as the case should be.

Finally in figures 6(a) \& (b) we draw the values of $C V a R_{P, \Delta L}, r_{P, \Delta L}$ and $\operatorname{VaR}_{P, \Delta L}$ for the deterministic unequal weight optimized problem considering the in-sample and out-ofsample examples respectively. It is true that $\operatorname{VaR}_{P, \Delta L}$ values change only with $\alpha_{P, \Delta L}$, but what is note-worthy is the corresponding marked fluctuation in $C V a R_{P, \Delta L}$ values which are much more pronounced than the corresponding values depicted in figures 5(a) \& (b). The logical reason for such high values of $C V a R_{P, \Delta L}$ may be the fact that we first optimize the weights of the indices/financial scripts/assets and then obtain $C V a R_{P, \Delta L}$ for the unequal weight problem. These values of $C V a R_{P, \Delta L}$ are definitely optimal (with respect to minimization) and better than the sub-optimal values obtained for the deterministic equal weight model, where the weights are already pre-fixed and not optimized.

\section{Conclusion and future work}

The research work deals with a simple yet interesting application of RO in financial portfolio optimization. In our proposed model we consider an optimization problem whereby we use CVaR as the risk measure (as it eliminates the drawbacks of VaR) and the average return considering the loss distribution of 12 indices/financial scripts/assets. We find the robust counterpart of the two probabilistic constraints and run a whole set of exhaustive simulation runs considering 12 different indices/financial scripts/assets for both in-sample as well as out-of-sample data points with some interesting results.

A comparison of our work with existing research corroborates few important points which are:

1) Portfolios optimized using robust methods yield better combinations of risk and returns profiles, as the case should be, due to the fact that an investor is able to portray his/her risk level more precisely due to the uncertainty values of the input data.

2) Probability/reliability levels force diversification and hence reduces portfolio risk.

3) An increase in the level of probability/reliability helps better portfolio management, as it aids diversification. 
4) A decrease in investor's attitude of how probable/reliable the input data is, has an adverse effect on the optimal value of the portfolio risk.

The work may be extended to cases where we consider RO in other kind of financial optimization problems whereby one can use Quadratic Programming (QP), Integer Programming (IP), Semi-Definite Programming (SDP), Mixed Integer Programming (MIP), etc., depending on the complexity of the portfolio one wants to formulate. Possibility of improvement would also encompass areas where our main concern would be to develop solution methodology which can handle the robust counterparts of different optimization problems like those mentioned above. This can definitely be a good focus area of research, considering the fact that robust counterpart of simple linear program, which are conic optimization problem by their own right, are themselves, computationally expensive.

Furthermore our research work tries to capture the effect of extreme uncertainty of parameters, and we propose solutions that are robust with respect to extreme realization of parameter values. Our proposed model suggests the optimum values for portfolio CVaR, portfolio returns and $\mathrm{VaR}$ under extreme uncertainty in parameters values. Moreover as EVD of financial asset returns are considered to measure the tail risks, hence we consider robust formulations of portfolio optimization models using financial indices/financial scripts/assets which do have heavy tails.

\section{References}

[1] Adida, E. and Perakis, G., A robust optimization approach to dynamic pricing and inventory control with no backorders. Mathematical Programming, 107, 2006, 97 129.

[2] Atamtäurk, A., Strong formulations of Robust Mixed 0-1 programming. Mathematical Programming: Series (B), 108, 2006, 235-250.

[3] Atamtäurk, A. and Zhang, M., Two-stage Robust Network flow and Design under Demand Uncertainty. Operations Research, 55, 2007, 662-673.

[4] Ben-Tal, A., Bertsimas, D. and Brown, D. B., A Soft Robust Model for Optimization under Ambiguity. Operations Research, 58, 2010, 1220-1234.

[5] Ben-Tal, A., Boyd, S. and Nemirovski, A., Extending the scope of robust optimization. Mathematical Programming: Series (B), 107, 2006, 63-89.

[6] Ben-Tal, A., El Ghaoui, L. and Nemirovski, A., Robust semidefinite programming in Semi-definite programming and applications, R. Saigal, Vandenberghe, H. Wolkowicz (Edited), Kluwer Academic Publishers, 2000a.

[7] Ben-Tal, A., El-Ghaoui, L. and Nemirovski, A., Robust optimization: Princeton Series in Applied Mathematics. Princeton University Press, Princeton, NJ, 2009.

[8] Ben-Tal, A., Golany, B., Nemirovski, A. and Vial, J. P., Supplier-retailer flexible commitments contracts: A Robust Optimization Approach. Manufacturing and Service Operations Management, 7, 2005, 248-271.

[9] Ben-Tal, A., Goryashko, A., Guslitzer, E. and Nemirovski, A., Adjustable Robust solutions of uncertain linear Programs. Mathematical Programming, 99, 2004, 351376. 
[10] Ben-Tal, A., Margalit, T. and Nemirovski, A., Robust modeling of multi-stage portfolio problems; High Performance Optimization, Frenk, H., Roos, C., Terlaky, T., Zhang, S. (Edited), Kluwer Academic Publishers, 2000b, 303-328.

[11] Ben-Tal, A. and Nemirovski, A., Robust truss topology design via semi-definite programming. SIAM Journal on Optimization, 7, 1997, 991-1016.

[12] Ben-Tal, A. and Nemirovski, A., Robust convex optimization. Mathematics of Operations Research, 23, 1998, 769-805.

[13] Ben-Tal, A. and Nemirovski., A, Robust solutions to uncertain linear programs. Operations Research Letters, 25, 1999, 1-13.

[14] Ben-Tal, A. and Nemirovski, A., Robust solutions of Linear Programming problems contaminated with uncertain data. Mathematical Programming: Series (A). 88, 2000c, 411-424.

[15] Ben-Tal, A. and Nemirovski, A., Lectures on modern convex optimization: Analysis, algorithms and engineering applications, MPR-SIAM Series on Optimization, SIAM, Philadelphia, 2001a.

[16] Ben-Tal, A. and Nemirovski, A., On approximate Robust counterparts of uncertain semi-definite and conic quadratic programs. Proceedings of $20^{\text {th }}$ IFIP TC7 Conference on System Modelling and Optimization, July 23-27, Trier, Germany, 2001b.

[17] Ben-Tal, A. and Nemirovski, A., Robust optimization-Methodology and Applications. Mathematical Programming: Series (B), 92, 2002a, 453-480.

[18] Ben-Tal, A. and Nemirovski, A., On tractable approximations of uncertain linear matrix inequalities affected by interval uncertainty. SIAM Journal on Optimization, 12, 2002b, 811-833.

[19] Ben-Tal, A., Nemirovski, A. and Roos, C., Robust solutions of uncertain quadratic and conic-quadratic problems. SIAM Journal on Optimization, 13, 2002c, 535-560.

[20] Bertsekas, D. P., Dynamic Programming and Optimal Control (Volume: I). Athena Scientific, Belmont, Massachusetts, USA, 1995.

[21] Bertsimas, D. and Caramanis, C., Finite adaptability in Multistage Linear Optimization. IEEE Transactions on Automatic Control, 55, 2007, 2751-2766.

[22] Bertsimas, D. and Brown, David B., Constructing Uncertainty Sets for Robust Linear Optimization, Operations Research, 57, 2009, 1483-1495.

[23] Bertsimas, D., Brown, D. B. and Caramanis, C., Theory and Applications of Robust Optimization. SIAM Review, 53, 2011, 464-501.

[24] Bertsimas, D., Nohadani, O. and Teo, K. M., Robust Optimization for Unconstrained Simulation-based Problems. Operations Research, 58, 2010, 161178.

[25] Bertsimas, D., Pachamanova, D. and Sim, M., Robust linear optimization under general norms. Operations Research Letters, 32, 2004, 510-516.

[26] Bertsimas, D. and Popescu, I., Optimal inequalities in probability theory: A convex optimization approach. SIAM Journal of Optimization, 15, 2004, 780-800.

[27] Bertsimas, D. and Sim, M., Robust discrete optimization and network flows. Mathematical Programming: Series: Series (B), 98, 2003, 49-71.

[28] Bertsimas, D. and Sim, M., The price of robustness. Operations Research, 52, 2004, 35-53.

[29] Bertsimas, D. and Sim, M., Tractable approximations to robust conic optimization problems. Mathematical Programming: Series (B), 107, 2006, 5-36. 
[30] Bertsimas, D. and Thiele, A., A robust optimization approach to supply chain management. Operations Research, 54, 2006, 150-168.

[31] Bertsimas, D. and Tsitsiklis, J., Introduction to Linear Optimization. Athena Scientific, 1997.

[32] Beyer, H-G. and Sendhoff, B., Robust optimization-A comprehensive survey. Computer Methods in Applied Mechanics and Engineering, 196, 2007, 3190-3128.

[33] Bienstock, D, Histogram models for robust portfolio optimization. Journal of Computational Finance, 11, 2007, 1-64.

[34] Birge, J. R. and Louveaux, F., Introduction to Stochastic Programming. SpringerVerlag, 1997.

[35] Boyd, S., El Ghaoui, L., Feron, E. and Balakrishnan, V., Linear Matrix Inequalities in System and Control Theory. SIAM, 1994.

[36] Boyd, S., Kim, S. J., Patil, D. and Horowitz, M., Digital circuit sizing via geometric programming. Operations Research, 53, 2005, 899-932.

[37] Boyd, S. and Vandenberghe, L., Semidefinite programming. SIAM Review, 38, 1996, 49-95.

[38] Boyd, S. and Vandenberghe, L., Convex Optimization. Cambridge University Press, 2004.

[39] Chen, W. and Sim, M., Goal Driven Optimization. Operations Research. 57, 2009, 342-357.

[40] Chen, W., Sim, M., Sun, J. and Teo, Chung-Piaw, From CVaR to Uncertainty Set: Implications in Joint Chance Constrained Optimization, Operations Research, 58, 2010, 470-485.

[41] Chen, X., Sim, M. and Sun, P., A robust optimization perspective to stochastic programming. Operations Research, 55, 2007, 1058-1071.

[42] Chen, X., Sim, M., Sun, P. and Zhang, J., A linear-decision based approximation approach to stochastic programming. Operations Research, 56, 2008, 344-357.

[43] Chen, X. and Zhang, Y., Uncertain Linear Programs: Extended Affinely Adjustable Robust Counterparts. Operations Research, 57, 2009, 1469-1482.

[44] Dembo, R., Scenario optimization, Annals of Operations Research, 30, 1991, 63-80.

[45] Duzgun, R. and Thiele, A., Dynamic models of Robust optimization, in J. Cochran, editors, Encyclopedia of Operations Research and Management Science, Wiley, New York, 2011.

[46] El Ghaoui, L. and Lebret, H., Robust solutions to least-square problems with uncertain data matrices. SIAM Journal of Matrix Analysis and Applications, 18, 1997, 1035-1064.

[47] El Ghaoui, L., Oustry, F. and Lebret, H., Robust solutions to uncertain semi-definite programs. SIAM Journal on Optimization, 9, 1998, 33-52.

[48] El Ghaoui, L., Maksim Oks. and Oustry, F., Worst-Case Value-at-Risk and Robust Portfolio Optimization: A Conic Programming Approach. Operations Research, 51, 2003, 543-556.

[49] Fabozzi, Frank J., Huang, D. and Zhou, G., Robust portfolios: contributions from operations research and finance, Annals of Operations Research, 176, 2010, 191220.

[50] Fabozzi, Frank J., Kolm, P. N., Pachamanova, D. A. and Focardi, S. M, Robust Portfolio Optimization. Journal of Portfolio Management, 33, 2007, 40-48. 
[51] Goldfarb, D. and Iyengar, G., Robust Portfolio Selection Problems. Mathematics of Operation Research, 28, 2003, 1-38.

[52] Gotoh, J, Shinozaki, K. and Takeda, A., Robust Portfolio Techniques for Mitigating the Fragility of CVaR Minimization and Generalization to Coherent Risk Measures, Quantitative Finance, 13, 2013, 1621-1635.

[53] Halldórsson, B. V. and Tütüncü, R. H., An interior-point method for a class of saddle point problems. Journal of Optimization Theory and Applications, 116, 2003, 559-590.

[54] Huang, D., Zhu, Shu-Shang., Fabozzi, Frank J. and Fukushima, M., Portfolio selection with uncertain exit time: A robust CVaR approach, Journal of Economic Dynamics and Control, 32, 2008, 594-623.

[55] Iyengar, G., Robust dynamic programming. Mathematics of Operations Research, 30, 1994, 257-280.

[56] Kall, P. and Wallace, S., Stochastic Programming. John Wiley \& Sons, 1994.

[57] Kouvelis, P. and Yu, G., Robust discrete optimization and its applications. Kluwer Academic Publishers, Norwell, MA, 1997.

[58] Lahiri, S. N., On the moving block bootstrap under long range dependence, Statistics and Probability Letters. 18, 1993, 405-413.

[59] Lahiri, S. N., Resampling methods for dependent data, Springer, New York, 2003.

[60] Markowitz, H., Portfolio Selection, The Journal of Finance, 7, 1952, 77-91.

[61] Mulvey, J., Vanderbei, R. and Zenios, S., Robust optimization of large-scale systems. Operations Research, 43, 1995, 264-281.

[62] Nemhauser, G. L. and Wolsey, L. A.,. Integer and Combinatorial Optimization. Wiley-Interscience Series in Dicrete Mathematics and Optimization, USA, 1988.

[63] Pinar, M. and Tütüncü, R., Robust profit opportunities in risky financial portfolios, Operations Research Letters, 33, 2005, 331-340.

[64] Prékopa, A., Stochastic Programming. Kluwer, 1995.

[65] Shapiro, A., On complexity of multistage stochastic programs. Operations Research Letters, 34, 2006, 1-8.

[66] Sim, M. and Goh, J., Robust Optimization Made Easy with ROME. Operations Research, 59, 2011, 973-985.

[67] Sniedovich, M., The art and science of modeling decision-making under severe uncertainty, Decision Making in Manufacturing and Services, 1, 2007, 111-136.

[68] Soyster, A. L., Convex programming with set-inclusive constraints and applications to inexact linear programming. Operations Research, 21, 1973, 1154-1157.

[69] Tütüncü, R. H. and Koenig, M., Robust asset allocation. Annals of Operations Research, 132, 2004, 157-187.

[70] Zhou, K., Doyle, J. C. and Glove, K., Robust and Optimal Control. Prentice-Hall, NJ, 1996.

[71] Zhu, S. and Fukushima, M., Worst-Case Conditional Value-at-Risk with Application to Robust Portfolio Management, Operations Research, 57, 2009, 1155-1168.

Received 31.05.2015, Accepted 23.01.2016 


\section{Appendix A.1: Proof of Theorem 1}

If $\boldsymbol{x}=\left(x_{1}, \ldots, x_{N}\right), \boldsymbol{z}=\left(z_{1}, \ldots, z_{N}\right)$ and $\boldsymbol{w}=\left(w_{1}, \ldots, w_{N}\right)$, are feasible for (4) and (5), then $\boldsymbol{x}$ is feasible for constraint $\operatorname{Pr}\left[\left\{\frac{1}{\left(1-\alpha_{\Delta L}\right) T} \sum_{i=1}^{T} \sum_{j=1}^{N}\left(r_{j, i} x_{j}-\gamma_{\Delta L, P}\right)^{+}+\gamma_{\Delta L, P}\right\} \leq\right.$ $\left.\operatorname{CVaR}_{P, \Delta L}\right] \geq \beta_{1}$ with probability at least $\left\{1-\exp \left(-\frac{\Omega_{1}^{2}}{2}\right)\right\}$. Now the constraint $\operatorname{Pr}\left[\left\{\frac{1}{\left(1-\alpha_{\Delta L}\right) T} \sum_{i=1}^{T} \sum_{j=1}^{N}\left(r_{j, i} x_{j}-\gamma_{\Delta L, P}\right)^{+}+\gamma_{\Delta L, P}\right\} \leq \operatorname{CVaR}_{P, \Delta L}\right] \geq \beta_{1}$ can be written in the form of perturbation set as

$\operatorname{Pr}\left[\left[\left\{\frac{1}{\left(1-\alpha_{\Delta L}\right) T}\left(\sum_{i=1}^{T} \sum_{j=1}^{N} \bar{r}_{j, i} x_{j}+\sum_{i=1}^{T} \sum_{j=1}^{N} \zeta_{j}\left[\sigma_{j}\right]^{T} x_{j}\right)-\gamma_{\Delta L, P}\right\}^{+}+\gamma_{\Delta L, P}\right] \leq C V a R_{P, \Delta L}\right] \geq \beta_{1}$

If one considers the term inside the probability in (A.1.1), then one can write it as

$\sum_{i=1}^{T} \sum_{j=1}^{N} \zeta_{j}\left[\sigma_{j}\right]^{T} x_{j} \leq\left(\operatorname{CVaR}_{P, \Delta L}-\gamma_{\Delta L, P}\right) \times\left(1-\alpha_{\Delta L}\right) T+\gamma_{\Delta L, P}-\sum_{i=1}^{T} \sum_{j=1}^{N} \bar{r}_{j, i} x_{j}($ A.1.2)

Moreover we are aware of the general uncertain linear inequality which is of the form as given below:

$\boldsymbol{a}^{T} \boldsymbol{x} \leq \boldsymbol{b}, \quad[\boldsymbol{a} ; \boldsymbol{b}]=\left[\boldsymbol{a}^{0} ; \boldsymbol{b}\right]+\sum_{j=1}^{N} \zeta_{j}\left[a^{j} ; \mathbf{0}\right]$, i.e.,

$$
\sum_{j=1}^{N} \zeta_{j}\left[a^{j}\right]^{T} \boldsymbol{x} \leq \boldsymbol{b}-\left[\boldsymbol{a}^{0}\right]^{T} \boldsymbol{x}
$$

Here (i) $\boldsymbol{a}^{0}$ is the vector set of nominal values of uncertain coefficients, (ii) $\boldsymbol{b}$ is the deterministic vector signifying the right hand side of the constraints and (iii) $a^{j}, j=$ $1,2, \ldots, N$ denotes the basic shifts of the $j^{\text {th }}$ coefficient.

A comparison of (A.1.2) and (A.1.3) gives us,

$$
\begin{gathered}
{\left[a^{j}\right]^{T} \boldsymbol{x}=\left[\sigma_{j}\right]^{T} x_{j}} \\
\boldsymbol{b}=\left(C V a R_{P, \Delta L}-\gamma_{\Delta L, P}\right) \times\left(1-\alpha_{\Delta L}\right) T \\
{\left[\boldsymbol{a}^{0}\right]^{T} \boldsymbol{x}=\sum_{i=1}^{T} \sum_{j=1}^{N} \bar{r}_{j, i} x_{j}}
\end{gathered}
$$

Box Robustness: In case of Box Robustness, or Interval Uncertainty, $Z$ (i.e., the uncertainty set) corresponds to a Box of the form $Z=B o x_{1}=\left\{\zeta \in \mathbb{R}^{N}:\|\zeta\|_{\infty} \leq 1\right\}$. Utilizing the concept of Box robustness, (A.1.3) can be written as $\sum_{j=1}^{N} \zeta_{j}\left[a^{j}\right]^{T} \boldsymbol{x} \leq \boldsymbol{b}-$ $\left[\boldsymbol{a}^{0}\right]^{T} \boldsymbol{x}, \forall\left(\boldsymbol{\zeta}:\left|\zeta_{j}\right| \leq 1, j=1,2, \ldots \ldots, N\right)$. Furthermore considering maximum perturbation value of the uncertain variable, $\zeta_{j}$, we have $\max _{-1 \leq \zeta_{j} \leq+1}\left[\sum_{j=1}^{N} \zeta_{j}\left[a^{j}\right]^{T} \boldsymbol{x}\right] \leq \boldsymbol{b}-\left[\boldsymbol{a}^{0}\right]^{T} \boldsymbol{x}$.

Now the maximum value for the left hand side of the above given constraint is $\sum_{j=1}^{N}\left|\left[a^{j}\right]^{T} \boldsymbol{x}\right|$, so it can be written in the form of explicit convex constraint as follows:

$$
\left[\boldsymbol{a}^{0}\right]^{T} \boldsymbol{x}+\sum_{j=1}^{N}\left|z_{j}\right| \leq \boldsymbol{b}
$$

where $z_{j}, j=1, \ldots \ldots, N$ are deterministic dummy variables such that $-z_{j} \leq\left[a^{j}\right]^{T} \boldsymbol{x} \leq$ $z_{j}$ for $j=1,2, \ldots, N$ 
Ball Robustness: For the case when we have Ball Robustness, $Z$ corresponds to an ellipsoid. So the uncertainty set is ellipsoidal uncertainty set and we assume that $Z$ is only a ball of radius $\Omega_{1}$ whose centre is at origin and is of the form $Z=$ Ball $_{\Omega_{1}}=\{\zeta \in$ $\left.\mathbb{R}^{N}:\|\zeta\|_{2} \leq \Omega_{1}\right\}$.

Utilizing the concept of Ball robustness, (A.1.3) can be written as $\sum_{j=1}^{N} \zeta_{j}\left[a^{j}\right]^{T} \boldsymbol{x} \leq$ $\boldsymbol{b}-\left[\boldsymbol{a}^{0}\right]^{T} \boldsymbol{x}, \forall\left(\boldsymbol{\zeta}:\|\zeta\|_{2} \leq \Omega_{1}\right)$.

In a similar line (as for Box robustness), considering maximum perturbation value of the uncertain variable, $\zeta_{j}$, we have $\max _{\|\zeta\|_{2} \leq \Omega_{1}}\left[\sum_{j=1}^{N} \zeta_{j}\left[a^{j}\right]^{T} \boldsymbol{x}\right] \leq \boldsymbol{b}-\left[\boldsymbol{a}^{0}\right]^{T} \boldsymbol{x}$, hence the chance constraint (A.1.3) is of the following form given below:

$$
\operatorname{Pr}\left\{\sum_{j=1}^{N} \zeta_{j}\left[a^{j}\right]^{T} \boldsymbol{x} \leq \boldsymbol{b}-\left[\boldsymbol{a}^{0}\right]^{T} \boldsymbol{x}\right\} \geq \beta_{1}
$$

Now for (A.1.6) we are aware that the random variable $\zeta_{j}$ has $E\left\{\zeta_{j}\right\}=0$ and $\left\{\zeta_{j}\right\}_{j=1}^{N}$ are i.i.d., which results in $\boldsymbol{\Phi} \equiv \sum_{j=1}^{N} \zeta_{j}\left[a^{j}\right]^{T} \boldsymbol{x} \leq \boldsymbol{b}-\left[\boldsymbol{a}^{0}\right]^{T} \boldsymbol{x}$. Furthermore using the given properties of $\zeta_{j}$, which are $E\left\{\zeta_{j}\right\}=0$ and $\left\{\zeta_{j}\right\}_{j=1}^{N}$ being i.i.d., if $\boldsymbol{x}$ is fixed, we see that $\boldsymbol{\Phi}$ is a random variable with zero mean and a standard deviation given by $S D[\boldsymbol{\Phi}]=$ $\sqrt{\sum_{j=1}^{N}\left(\left[a^{j}\right]^{T} \boldsymbol{x}\right)^{2} E\left\{\zeta_{j}^{2}\right\}} \leq \sqrt{\sum_{j=1}^{N}\left(\left[a^{j}\right]^{T} \boldsymbol{x}\right)^{2}}$. Moreover for chance constraint (A.1.6) to be satisfied with probability $\geq \beta_{1}$ one easily notes that $\boldsymbol{\Phi}$ is never greater than the quantity $\Omega_{1} \sqrt{\sum_{j=1}^{N}\left(\left[a^{j}\right]^{T} \boldsymbol{x}\right)^{2}}$, where $\Omega_{1}$ is a safety parameter pertaining to $\beta_{1}$. Larger values of $\Omega_{1}$ implies less are chances for the constraint to violate the robust conditions. Here $\left[a^{j}\right]^{T}$ value is calculated from the extreme value distribution.

So the parametric safe versions of the randomly perturbed constraint are:

$$
\begin{aligned}
& \Omega_{1} \sqrt{\sum_{j=1}^{N}\left(\left[a^{j}\right]^{T} \boldsymbol{x}\right)^{2}} \leq \boldsymbol{b}-\left[\boldsymbol{a}^{0}\right]^{T} \boldsymbol{x}, \\
& {\left[\boldsymbol{a}^{0}\right]^{T} \boldsymbol{x}+\Omega_{1} \sqrt{\sum_{j=1}^{N}\left(\left[a^{j}\right]^{T} \boldsymbol{x}\right)^{2}} \leq \boldsymbol{b}}
\end{aligned}
$$

It is apparent that with properly defined $\Omega_{1}$ every feasible solution to this constraint satisfies the inequality (A.1.3) with probability at least $\beta_{1}$. Hence with $w_{j}, j=1,2, \ldots \ldots, N$ as deterministic coefficients and $\zeta_{j}, j=1,2, \ldots \ldots, N$ as independent random variable with zero mean and standard deviation $\Omega_{2} \sqrt{\sum_{j=1}^{N}\left(\left[a^{j}\right]^{T} \boldsymbol{x}\right)^{2}}$, for every $\Omega_{2} \geq 0$, it holds that

$$
\begin{aligned}
& \operatorname{Pr}\left\{\left[\boldsymbol{a}^{0}\right]^{T} \boldsymbol{x}+\sum_{j=1}^{N} \zeta_{j}\left[a^{j}\right]^{T} \boldsymbol{x} \geq \boldsymbol{b}\right\} \leq 1-\beta_{1} \\
& \quad \text { i.e., } \operatorname{Pr}\left\{\sum_{j=1}^{N} w_{j} \zeta_{j} \geq \Omega_{1} \sqrt{\sum_{j=1}^{N} w_{j}^{2}}\right\} \geq 1-\beta_{1} \text {, where, } \beta_{1}=1-\exp \left\{-\Omega_{1}^{2} / 2\right\}
\end{aligned}
$$

Ball-Box Robustness: Consider $z_{j}, w_{j}, j=1,2, \ldots \ldots \ldots N$ as the deterministic dummy variables and $\zeta_{j}, j=1,2, \ldots \ldots \ldots N$ with Box-Ball perturbations. Then using both $\|\zeta\|_{\infty} \leq 1$ and $\|\zeta\|_{2} \leq \Omega_{1}$ conditions along with the inequalities (A.1.5) and (A.1.7) we obtain $\left[\boldsymbol{a}^{0}\right]^{T} \boldsymbol{x}+\sum_{N}\left|z_{j}\right|+\Omega_{1} \sqrt{\sum_{j=1}^{N} w_{j}^{2}} \leq \boldsymbol{b}$ and $z_{j}+w_{j}=\left[a^{j}\right]^{T} \boldsymbol{x}$. 
Putting values of $\left[\boldsymbol{a}^{0}\right]^{T} \boldsymbol{x},\left[a^{j}\right]^{T} \boldsymbol{x}$ and $\boldsymbol{b}$ from (A.1.4) in the above equations we derive

$$
\begin{aligned}
& {\left[\left\{\frac{1}{\left(1-\alpha_{\Delta L}\right) T} \sum_{i=1}^{T} \sum_{j=1}^{N}\left(\bar{r}_{j, i} x_{j}+\sum_{N}\left|z_{j}\right|+\Omega_{1} \sqrt{\left.\left.\sum_{j=1}^{N} w_{j}^{2}-\gamma_{\Delta L, P}\right)^{+}\right\}}\right\}^{+} \gamma_{\Delta L, P}\right] \leq \operatorname{CVaR}_{P, \Delta L}\right.} \\
& \quad z_{j}+w_{j}=\sum_{j=1}^{N}\left[\sigma_{j}\right]^{T} x_{j}, \quad j=1, \ldots \ldots \ldots, N \\
& \text { where, } \beta_{1}=1-\exp \left\{-\Omega_{1}^{2} / 2\right\}
\end{aligned}
$$

\section{Appendix A.2: Proof of Theorem 2}

If $\boldsymbol{x}=\left(x_{1}, \ldots, x_{N}\right), \boldsymbol{z}=\left(z_{1}, \ldots, z_{N}\right), \boldsymbol{w}=\left(w_{1}, \ldots, w_{N}\right)$, is feasible for (6) and (7), then $\boldsymbol{x}$ is feasible for constraint $\operatorname{Pr}\left[\left\{\frac{1}{T} \sum_{i=1}^{T} \sum_{j=1}^{N} r_{j, i} x_{j}\right\} \geq r_{P, \Delta L}\right] \geq \beta_{2}$ with probability at least $\left\{1-\exp \left(-\frac{\Omega_{2}^{2}}{2}\right)\right\}$.

$\operatorname{Pr}\left[\left\{\frac{1}{T}\left(\sum_{i=1}^{T} \sum_{j=1}^{N} \bar{r}_{j, i} x_{j}-\sum_{i=1}^{T} \sum_{j=1}^{N} \zeta_{j}\left[\sigma_{j}\right]^{T} x_{j}\right)\right\} \geq r_{P, \Delta L}\right] \geq \beta_{2}$

If one considers the term inside the probability in (A.2.1), then one can write it as

$\left.-\sum_{i=1}^{T} \sum_{j=1}^{N} \zeta_{j}\left[\sigma_{j}\right]^{T} x_{j}\right) \geq r_{P, \Delta L} \times T-\sum_{i=1}^{T} \sum_{j=1}^{N} \bar{r}_{j, i} x_{j}$

Moreover we are aware of the general uncertain linear inequality which is of the form as given below:

$$
\begin{aligned}
\boldsymbol{a}^{T} \boldsymbol{x} \geq \boldsymbol{b}, \quad[\boldsymbol{a} ; \boldsymbol{b}]=\left[\boldsymbol{a}^{0} ; \boldsymbol{b}\right]- & \sum_{j=1}^{N} \zeta_{j}\left[a^{j} ; 0\right], \text { i.e., } \\
& -\sum_{j=1}^{N} \zeta_{j}\left[a^{j}\right]^{T} \boldsymbol{x} \geq \boldsymbol{b}-\left[\boldsymbol{a}^{0}\right]^{T} \boldsymbol{x}
\end{aligned}
$$

Here (i) $\boldsymbol{a}^{0}$ is the vector set of nominal values of uncertain coefficients, (ii) $\boldsymbol{b}$ is the deterministic vector signifying the right hand side of the constraints and (iii) $a^{j}, j=$ $1,2, \ldots, N$ denotes the basic shifts of the $j^{\text {th }}$ coefficient.

A comparison of (A.2.2) and (A.2.3) gives us

$$
\begin{gathered}
{\left[a^{j}\right]^{T} \boldsymbol{x}=\left[\sigma_{j}\right]^{T} x_{j}} \\
\boldsymbol{b}=r_{P, \Delta L} \times T \\
{\left[\boldsymbol{a}^{0}\right]^{T} \boldsymbol{x}=\sum_{i=1}^{T} \sum_{j=1}^{N} \bar{r}_{j, i} x_{j}}
\end{gathered}
$$

Box Robustness: In case of Box Robustness, or Interval Uncertainty, $Z$ (i.e., the uncertainty set) corresponds to a Box of the form $Z=B o x_{1}=\left\{\zeta \in \mathbb{R}^{N}:\|\zeta\|_{\infty} \leq 1\right\}$. Now utilizing the concept of $\boldsymbol{B o x}$ robustness, (A.2.3) can be written as $-\sum_{j=1}^{N} \zeta_{j}\left[a^{j}\right]^{T} \boldsymbol{x} \geq \boldsymbol{b}-$ $\left[\boldsymbol{a}^{0}\right]^{T} \boldsymbol{x}, \forall\left(\zeta:\left|\zeta_{j}\right| \leq 1, j=1, \ldots \ldots, N\right)$. Furthermore considering maximum perturbation value of the uncertain variable, $\zeta_{j}$, we have $\max _{-1 \leq \zeta_{j} \leq+1}\left[-\sum_{j=1}^{N} \zeta_{j}\left[a^{j}\right]^{T} \boldsymbol{x}\right] \geq \boldsymbol{b}-\left[\boldsymbol{a}^{0}\right]^{T} \boldsymbol{x}$.

Now the maximum value for the left hand side of the above given constraint is $\sum_{j=1}^{N}\left|\left[a^{j}\right]^{T} \boldsymbol{x}\right|$, so the above can be written in the form of explicit convex constraint as follows: 


$$
\left[\boldsymbol{a}^{0}\right]^{T} \boldsymbol{x}-\sum_{j=1}^{N}\left|z_{j}\right| \geq \boldsymbol{b}
$$

Where $z_{j}, j=1, \ldots \ldots, N$ are deterministic dummy variables such that $-z_{j} \leq\left[a^{j}\right]^{T} x \leq$ $z_{j}, j=1, \ldots, N$

Ball Robustness: For the case when we have Ball Robustness, $Z$ corresponds to an ellipsoid. So the uncertainty set is ellipsoidal uncertainty set, hence we assume that $Z$ is only a ball of radius $\Omega_{1}$ whose centre is at origin and is of the form $Z=$ Ball $_{\Omega_{2}}=$ $\left\{\zeta \in \mathbb{R}^{N}:\|\zeta\|_{2} \leq \Omega_{2}\right\}$.

Utilizing the concept of Ball robustness, (A.2.3) can be written as $-\sum_{j=1}^{N} \zeta_{j}\left[a^{j}\right]^{T} \boldsymbol{x} \geq$ $\boldsymbol{b}-\left[\boldsymbol{a}^{0}\right]^{T} \boldsymbol{x}, \forall\left(\zeta:\|\zeta\|_{2} \leq \Omega_{2}\right)$.

In a similar line (as for Box robustness), considering maximum perturbation value of the uncertain variable, $\zeta_{j}$, we have $\max _{\|\zeta\|_{2} \leq \Omega_{2}}\left[-\sum_{j=1}^{N} \zeta_{j}\left[a^{j}\right]^{T} \boldsymbol{x}\right] \geq \boldsymbol{b}-\left[\boldsymbol{a}^{0}\right]^{T} \boldsymbol{x}$, hence the chance constraint (A.2.3) is of the following form given below: :

$$
\operatorname{Pr}\left\{-\sum_{j=1}^{N} \zeta_{j}\left[a^{j}\right]^{T} \boldsymbol{x} \geq \boldsymbol{b}-\left[\boldsymbol{a}^{0}\right]^{T} \boldsymbol{x}\right\} \geq \beta_{2}
$$

Now for (A.2.6) we are aware that the random variable $\zeta_{j}$ has $E\left\{\zeta_{j}\right\}=0$ and $\left\{\zeta_{j}\right\}_{j=1}^{N}$ are i.i.d., which results in $\boldsymbol{\Psi} \equiv-\sum_{j=1}^{N} \zeta_{j}\left[a^{j}\right]^{T} \boldsymbol{x} \geq \boldsymbol{b}-\left[\boldsymbol{a}^{0}\right]^{T} \boldsymbol{x}$. Furthermore using the given properties of $\zeta_{j}$, which are $E\left\{\zeta_{j}\right\}=0$ and $\left\{\zeta_{j}\right\}_{j=1}^{N}$ being i.i.d., if $\boldsymbol{x}$ is fixed, we see that $\boldsymbol{\Psi}$ is a random variable with zero mean and a standard deviation given by $S D[\Psi]=$ $\sqrt{\sum_{j=1}^{N}\left(\left[a^{j}\right]^{T} \boldsymbol{x}\right)^{2} E\left\{\zeta_{j}^{2}\right\}} \leq \sqrt{\sum_{j=1}^{N}\left(\left[a^{j}\right]^{T} \boldsymbol{x}\right)^{2}}$. Moreover for the chance constraint (A.2.6) to be satisfied with probability $\geq \beta_{2}$ one easily notes that $\boldsymbol{\Psi}$ is never greater than the quantity $-\Omega_{2} \sqrt{\sum_{j=1}^{N}\left(\left[a^{j}\right]^{T} \boldsymbol{x}\right)^{2}}$, where $\Omega_{2}$ is a safety parameter pertaining to $\beta_{2}$. Larger values of $\Omega_{2}$ implies less are chances for the constraint to violate the robust conditions. Here, $\left[a^{j}\right]^{T}$ comes from extreme value distribution.

So the parametric safe versions of the randomly perturbed constraint are:

$$
\begin{gathered}
-\Omega_{2} \sqrt{\sum_{j=1}^{N}\left(\left[a^{j}\right]^{T} \boldsymbol{x}\right)^{2}} \geq \boldsymbol{b}-\left[\boldsymbol{a}^{0}\right]^{T} \boldsymbol{x}, \\
{\left[\boldsymbol{a}^{0}\right]^{T} \boldsymbol{x}-\Omega_{2} \sqrt{\sum_{j=1}^{N}\left(\left[a^{j}\right]^{T} \boldsymbol{x}\right)^{2}} \geq \boldsymbol{b}}
\end{gathered}
$$

It is apparent that with properly defined $\Omega_{2}$; every feasible solution to this constraint satisfies the inequality (A.2.3) with probability at least $\beta_{2}$. Hence with $w_{j}, j=1,2, \ldots \ldots, N$ as deterministic coefficients and $\zeta_{j}, j=1,2, \ldots \ldots, N$ as independent random variable with zero mean and standard deviation $\Omega_{2} \sqrt{\sum_{j=1}^{N}\left(\left[a^{j}\right]^{T} \boldsymbol{x}\right)^{2}}$, then for every $\Omega_{2} \geq 0$, it holds that

$$
\begin{aligned}
& \operatorname{Pr}\left\{\left[\boldsymbol{a}^{0}\right]^{T} \boldsymbol{x}-\sum_{j=1}^{N} \zeta_{j}\left[a^{j}\right]^{T} \boldsymbol{x} \leq \boldsymbol{b}\right\} \geq 1-\beta_{2} \\
& \quad \operatorname{Pr}\left\{-\sum_{j=1}^{N} w_{j} \zeta_{j} \leq-\Omega_{2} \sqrt{\sum_{j=1}^{N} w_{j}^{2}}\right\} \geq 1-\beta_{2}, \text { where, } \beta_{2}=1-\exp \left\{-\Omega_{2}^{2} / 2\right\}
\end{aligned}
$$


Ball-Box Robustness: Consider $z_{j}, w_{j}, j=1,2, \ldots \ldots \ldots \ldots$ as the deterministic dummy variables and $\zeta_{j}, j=1,2, \ldots \ldots \ldots N$ with Box-Ball perturbations. Then using both $\|\zeta\|_{\infty} \leq 1$ and $\|\zeta\|_{2} \leq \Omega_{2}$ conditions along with the inequalities (A.2.5) and (A.2.7) we obtain

$$
\left[\boldsymbol{a}^{0}\right]^{T} \boldsymbol{x}-\sum_{N}\left|z_{j}\right|-\Omega_{2} \sqrt{\sum_{j=1}^{N} w_{j}^{2}} \geq \boldsymbol{b} \text { and } z_{j}+w_{j}=\left[a^{j}\right]^{T} \boldsymbol{x}
$$

Putting values of $\left[\boldsymbol{a}^{0}\right]^{T} \boldsymbol{x},\left[a^{j}\right]^{T} \boldsymbol{x}$ and $\boldsymbol{b}$ from (A.2.4) in above equations, we get

$$
\left[\frac{1}{T}\left(\sum_{i=1}^{T} \sum_{j=1}^{N} \bar{r}_{j, i} x_{j}-\sum_{N}\left|z_{j}\right|-\Omega_{2} \sqrt{\sum_{j=1}^{N} w_{j}^{2}}\right)\right] \geq r_{P, \Delta L}
$$

$z_{j}+w_{j}=\sum_{j=1}^{N}\left[\sigma_{j}\right]^{T} x_{j}, \quad j=1, \ldots \ldots \ldots, N$

where, $\beta_{2}=1-\exp \left\{-\Omega_{2}^{2} / 2\right\}$ 